\title{
Single-Look SAR Tomography as an Add-On to PSI for Improved Deformation Analysis in Urban Areas
}

\author{
Muhammad Adnan Siddique, Student Member, IEEE, Urs Wegmüller, Senior Member, IEEE, \\ Irena Hajnsek, Fellow, IEEE, and Othmar Frey, Member, IEEE
}

\begin{abstract}
Persistent scatterer interferometry (PSI) is in operational use for spaceborne synthetic aperture radar (SAR)-based deformation analysis. A limitation inherently associated with PSI is that, by definition, a persistent scatterer (PS) is a single dominant scatterer. Therefore, pixels containing signal contributions from multiple scatterers, as in the case of a layover, are typically rejected in the PSI processing, which in turn limits deformation retrieval. SAR tomography has the ability to resolve layovers. This paper investigates the added value that can be achieved by operationally combining SAR tomography with a PSI approach toward the objective of improving deformation sampling in layover-affected urban areas. Different tomographic phase models are implemented and compared as regards their suitability in resolving layovers. Single-look beamforming-based tomographic inversion and a generalized likelihood ratio test (GLRT)-based detection strategy are used to detect single and double scatterers. The quantity of the detected scatterers is weighed against their quality as defined in terms of the phase deviation between the single-look complex (SLC) measurements and the tomographic model fit. The gain in deformation sampling that can be derived with tomography relative to a PSI-based analysis is quantitatively assessed, and alongside the quality of the scatterers obtained with tomography is compared with the quality of the PSs identified with a PSI approach. The experiments are performed on an interferometric stack of 50 TerraSAR-X stripmap images. The results obtained show that, although there is a tradeoff between the quantity and the quality of the detected scatterers, the tested SAR tomography approach leads to an improvement in deformation sampling in layover-affected areas.
\end{abstract}

Index Terms-Deformation analysis in urban areas, persistent scatterer interferometry (PSI), synthetic aperture radar (SAR) tomography.

Manuscript received December 28, 2015; revised March 21, 2016 and April 25, 2016; accepted May 11, 2016. Date of publication July 9, 2016; date of current version August 11, 2016. This work was supported by the Swiss Space Office, State Secretariat for Education, Research, and Innovation of the Swiss Confederation (SERI/SSO) in the frame of the "Space Technology Studies" MdP2012 project "GAMMA software module for spaceborne SAR tomography."

M. A. Siddique is with the Chair of Earth Observation and Remote Sensing, Institute of Environmental Engineering, Swiss Federal Institute of Technology, 8093 Zurich, Switzerland (e-mail: siddique@ifu.baug.ethz.ch).

U. Wegmüller is with GAMMA Remote Sensing AG, 3073 Gümligen, Switzerland (e-mail: wegmuller@gamma-rs.ch).

I. Hajnsek is with the Chair of Earth Observation and Remote Sensing, Institute of Environmental Engineering, Swiss Federal Institute of Technology, 8093 Zurich, Switzerland, and also with the Microwaves and Radar Institute, German Aerospace Center, 82234 Wessling, Germany (e-mail: hajnsek@ifu.baug.ethz.ch).

O. Frey is with the Chair of Earth Observation and Remote Sensing, Institute of Environmental Engineering, Swiss Federal Institute of Technology, 8093 Zurich, Switzerland, and also with GAMMA Remote Sensing AG, 3073 Gümligen, Switzerland (e-mail: otfrey@ethz.ch/frey@gamma-rs.ch).

Color versions of one or more of the figures in this paper are available online at http://ieeexplore.ieee.org.

Digital Object Identifier 10.1109/TGRS.2016.2581261

\section{INTRODUCTION}

$\mathbf{M}$ ULTITEMPORAL synthetic aperture radar (SAR) interferometric techniques are widely used to monitor surface deformation caused by various geophysical processes (natural as well as anthropogenic), such as tectonic and volcanic activities [1]-[3], mass movements on unstable slopes [4], [5], and mining and groundwater pumping [6]-[9]. The dynamics of such processes are sampled with time series of interferometric SAR data. Differential interferometric SAR (DInSAR) [10], [11] methods exploit the temporal baselines among one or more interferograms to extract the phase components correlated with scatterer motion. The performance of these methods is limited by the presence of unwanted phase components, such as the atmospheric phase screen (APS), and phase degradation caused by temporal and geometric decorrelations. Persistent scatterer interferometry (PSI) [12]-[16] is an advanced DInSAR concept that circumvents these limitations by observing the phase histories of the so-called persistent scatterers (PSs) in a relatively large stack of interferograms. These are single dominant pointlike scatterers marked by high temporal coherence across the entire stack. Since they are less susceptible to the decorrelation phenomena, their unknown motion parameters can be more reliably estimated. A large quantity of PSs is generally desired to effectively retrieve information about the observed geophysical process.

In the context of long-term monitoring of deformation in urban areas, PSI has proven to be an invaluable tool, offering millimeter-scale precision [17], [18] for extended areas of observation. Man-made structures, such as rooftops, railways, metallic lamp posts, and window panes, are good PS candidates (PSCs). A PSI analysis of an urban area generally reveals a sizable number of PSs, particularly with high-resolution spotlight images [19]. However, an inherent limitation associated with PSI is that, by definition, a PS is a single dominant scatterer within a range-azimuth resolution cell. Therefore, pixels containing backscatter of comparable energy from multiple scatterers, which may individually exhibit point-like behavior, are rejected. This situation arises often in layovers. Urban areas typically have buildings of different heights, and layovers such as those between the ground and the facade of a nearby building, or the rooftop of one building and the facade of a higher building in proximity, occur ubiquitously. A local PSI analysis of such buildings may suffer from poor deformation sampling due to the rejection of such layovers. To overcome this limitation, a higher order analysis [20] (i.e., considering the possibility of more than one scatterer in a range-azimuth pixel) is required. 
SAR tomography [21]-[30] is a multibaseline interferometric technique that allows higher order modeling using both the phase and amplitude of the backscatter. It serves as a means to separate individual scatterers in layover, which motivates its use as an add-on to PSI. While the classical use of SAR tomography has been the retrieval of reflectivity profile along the elevation (perpendicular to the line of sight (LOS) direction), the more advanced tomographic techniques simultaneously allow modeling the motion parameters of one or more scatterers in addition to their elevation. Notwithstanding that PSI and tomography may share the same phase models, whereas the former can retrieve the elevation (as residual topography) and motion parameters for a single scatterer only, the latter allows it for multiple scatterers in a given range-azimuth pixel. This paper investigates the added value that can be derived by the combined use of SAR tomographic techniques and PSI, particularly toward the objective of extending deformation analysis to layover-affected areas.

\section{A. Related Work}

The early developments in SAR tomography focused on exploiting the spatial baselines of the data stack to build a synthetic aperture in the elevation, thereby extending the conventional 2-D SAR imaging to 3-D. The scatterers were assumed stationary. The concept of differential tomography, as introduced in [31] and [32], raised interest in using tomographic techniques for deformation analysis as well. Differential tomography is a means to simultaneously model scatterer elevation and deformation. It exploits both the spatial and temporal baselines at the same time. The deformation is assumed temporally linear. Results on real data have been presented in various studies [26], [33]-[38]. As reported in some PSI investigations with X-band data, such as [17], [19], [39], and [40], scatterers in urban areas may additionally be subject to nonlinear motion due to thermal dilation. The phase model needs to be extended to account for the phase variations associated with nonlinear motion. In [41] and [42], the extended phase model considers the nonlinear motion as a temporally sinusoidal variation alongside the linear component. The investigations presented in [43]-[45], however, explicitly include local temperature values to model phase variations related to thermal dilation; the results are provided for single scatterers only.

Different tomographic inversion methods have been proposed over time. In the case that the underlying signal model is assumed zero-mean circular Gaussian (fully developed speckle), multilooking is typically needed to estimate the sample covariance matrix and to allow for a reasonable inversion with spectral estimators such as CAPON [37], [46] and multiple signal classification (MUSIC) [47], [48]. Multilooking, however, reduces the spatial resolution in range/azimuth. The approach recently proposed in [49] is a multilook inversion method that uses a rather small degree of spatially adapted multilooking (averaging less than $<50$ pixels) to estimate the sample covariance matrix. The method uses the principal component analysis (PCA)-based interferometric stack filtering approach given in [50]. Layovers are resolved by associating the eigenvectors of the estimated covariance matrix with the different scattering mechanisms in the given (multilooked) pixel. With a slight compromise on spatial resolution, the method offers better coverage compared with single-look tomographic inversion. In [50], it is additionally reported that layover separation can be achieved without a priori phase calibration of the stack (as required for SAR tomography) since the approach does not require any assumption about the structure of the steering vector (for the given spatial and temporal baselines).

In case of quasi-deterministic scatterers with stable phase (speckle-free, point-like behavior), single-look inversion methods can be used. Among such methods are the standard beamforming (BF) [26], [34], [51] and singular value decomposition (SVD)-based techniques [30], [52]. BF is a simple and robust method, but it is susceptible to spectral leakage and high sidelobes. The resolution in elevation is determined by the extent and distribution of the spatial baselines and is at best limited to the Rayleigh resolution for the theoretical case of uniformly distributed baselines over the same extent. SVDbased techniques generally offer better sidelobe reduction and possibly a slight super-resolution, i.e., the resolution achieved can be slightly better than the Rayleigh limit. In the context of super-resolution, the single-look tomographic processing introduced in a compressive sensing (CS) signal reconstruction framework in [53] and [54] has been a significant advance. It is, however, computationally expensive. In [42], the overall computational expense of the framework is reduced by restricting the application of the CS-based reconstruction only to those pixels that contain closely spaced double scatterers classified a priori. The classification process uses informationtheoretic criteria (ITC), a trained support vector machine classifier and estimates of scatterer elevation and deformation parameters obtained with periodogram maximization (based on BF with amplitude-normalized single-look complex (SLC) pixel values). An alternative single-look method offering superresolution with relatively inexpensive computation has been recently proposed in [28]. It suggests interpolating the spatial baselines at virtual uniform locations, followed by tomographic inversion with MUSIC and CAPON beamforming (which also offer super-resolution in elevation).

Prior to tomographic inversion, a PSI analysis is typically needed to isolate the APS [55] and thereby phase calibrate the interferometric data stack. Another imperative step during tomographic processing is the estimation of the number of pointlike temporally coherent scatterers in a given range-azimuth pixel, i.e., model order selection. In [56], ITC-based model order selection methods have been discussed. In the case of tomographic focusing with parametric spectral estimators, e.g., nonlinear least squares [30], [48], MUSIC, etc., model order selection has to be applied a priori. With nonparametric estimators, such as BF, CAPON, or the SVD-based techniques, the number of scatterers superposed in a given pixel can be estimated a posteriori by observing the "maxima" [57] in the retrieved reflectivity profile. For the identification of single dominant scatterers (i.e., PSs), a detector based on the multiinterferogram complex coherence (MICC) has been used in [58] in the context of PSI processing. The MICC-based detector uses only the phase residuals (deviations between the differential 
phases and the phase model fit) neglecting the amplitude information. It has been shown in [34] that a generalized likelihood ratio test (GLRT)-based detection of single scatterers, which implicitly incorporates BF-based tomographic focusing and hence includes both the amplitude and phase information, improves performance both in terms of higher probability of detection for a given level of false alarm as well as higher accuracy of estimates. A "second order" version of MICC has been used in [20] for the detection of both single and double scatterers. A sequential use of GLRT has been theoretically developed and applied in [38] to decide among the hypotheses that a given pixel contains a single or double scatterer, or neither.

\section{B. Research Gaps}

Single-look SAR tomography can be combined with a PSI approach to improve deformation sampling in urban areas. Given the fact that PSI is nowadays operationally used for deformation analysis, a critical assessment of the utility offered by SAR tomography is required to justify its operational use as an add-on to PSI. Different aspects of a tomographic framework (signal modeling, inversion, scatter detection, etc.) need to be reviewed in this context and its performance compared with that of an established PSI approach using a common quality metric. The relative gain in deformation sampling with the added use of tomography, when using a typical SAR interferometric data stack (in terms of baselines, and spatial resolution), remains to be both quantitatively and qualitatively assessed.

\section{Contributions of This Paper}

To fill in the aforementioned research gaps, this paper provides the following main contributions.

1) Different phase models for tomography are analyzed in terms of their suitability for layover resolution and simultaneous estimation of deformation parameters.

2) The potential gain in deformation sampling using a tomographic approach in addition to a PSI approach is quantitatively discussed.

3) The quality of the scatterers obtained with tomography is compared with the quality of the PSs identified with a PSI approach (using a mutually consistent measure of quality).

\section{Outline}

First, we perform a PSI analysis for the selected urban area using the interferometric point target analysis (IPTA) [13] framework. A set of PSs is iteratively identified. The solution obtained includes the estimates of the residual topography, linear deformation, phase-to-temperature sensitivity, and the APS for each PS. Next, we implement BF-based tomographic inversion on the (phase calibrated) interferometric stack with three different phase models. The inversion is applied on all pixels, including those rejected in the PSI processing which potentially include double scatterers. The performance of the individual phase models, as regards the ability to resolve layovers, is evaluated. For the detection of single and double scatterers, we adopt a sequential GLRT strategy as proposed in [38]. Relative to the number of PSs identified separately in the PSI processing, we quantify the gain in deformation sampling owing to layover separations achieved with tomography. As a next step, we compare the quality of the scatterers obtained with tomography against the quality of the PSs identified in the PSI processing. Since a traditional PSI analysis uses only the phase information, we assess the quality of the PSs in terms of the residual phase, i.e., the root-mean-square (RMS) phase deviation between the differential phases and the phase model fit. Along similar lines, we evaluate the quality of the scatterers obtained with tomography on the basis of RMS phase deviation between the SLC measurements and the tomographic model fit. In this way, the quality metric used is mutually consistent and, therefore, allows for a performance comparison.

\section{E. Additional Remarks}

In case of single scatterers/PSs, it has been shown in [34] that a tomography/GLRT-based detection and estimation approach offers improved quality in comparison with the classical PSI/MICC-based approach. In this investigation, we conduct an extended (empirical) quality comparison that includes the case of double scatterers. We intend to comprehensively crossexamine the quality of the double scatterers against single scatterers obtained with tomography/GLRT at different detection thresholds and in turn relate it to the quality of the PSs identified separately with an IPTA-based PSI processing.

SAR tomographic analysis in urban areas has often been conducted with very high resolution $(\sim 1 \mathrm{~m})$ images acquired in spotlight imaging mode. The higher the resolution, the more is the tendency of the individual resolution cells towards pointlike scattering. Therefore, compared with stripmap images at $\sim 3 \mathrm{~m}$ resolution, spotlight images are more favorable for a PSI/tomographic analysis. Today, interferometric stacks of high-resolution spotlight-mode SAR data are available only for a very limited number of urban areas. Keeping in view this limitation, we perform this investigation on an interferometric stack comprising of stripmap images to assess the potential of tomography for a data type which is widely available and which is, therefore, more relevant in an operational scenario.

The remainder of this paper is organized as follows. Section II describes the methods used in this investigation for PSI processing, tomographic inversion, and scatterer detection. The characteristics of the interferometric data stack used in this investigation are given in Section III. Results are provided in Section IV, and a detailed discussion follows in Section V.

\section{Methods}

\section{A. PSI Processing Approach}

Prior to a tomographic analysis of an interferometric data stack, a PSI solution is generally needed to extract and remove the atmospheric phase contributions, thereby phase calibrating the stack. As per the objectives of this paper, we 
require a PSI solution to also serve as a reference against which we can compare the potential value addition (in terms of quantity and quality of the deformation samples) offered by the added use of single-look SAR tomography. In this context, we performed PSI processing using the IPTA [13] toolbox which supports SAR interferometric time-series analysis. The important aspects of the PSI processing in our case are as follows.

A reference layer was chosen from the stack of SLC SAR images, and all the layers in the stack were then geocoded using a multilooked intensity image of the reference [59], [60] and coregistered. An initial list of PSCs was prepared on the basis of high temporal stability of the backscattering and low spectral diversity. These two criteria act as a proxy to identify the pixels containing single point-like scatterers exhibiting longterm temporal coherence. As a next step, we generated point differential interferograms for the candidates, using the (single) reference scene. It was assumed that the unwrapped differential interferometric phase is a sum of the phase contributions from the residual topography, deformation, and the atmospheric path length delays. Exploiting the spatial and temporal baselines, we applied a 2-D least-squares regression to obtain an initial estimate of the residual topography $h$ and (average) linear deformation velocity $\nu$. We evaluated the quality of the estimates in terms of the RMS phase deviation $\sigma^{\text {ipta }}$ between the differential phases and the model fit. Low RMS phase deviation represents a better match with the phase model and hence indicates better quality. To ease computational burden of the processing, we curtailed the initial list of candidates using an adaptive point density reduction strategy [61]. The density of the candidates was reduced only in those local regions where it was too high (by masking preferentially the candidates of relatively low quality in the local neighborhood).

The APS behaves as a nuisance in the regression fitting. Assuming the APS to be spatially low frequency and temporally uncorrelated, we estimated it by spatial filtration and unwrapping of the phase residue in the neighborhood of the candidates that satisfied a quality criterion i.e., $\sigma^{\text {ipta }}$ below a certain preselected threshold. The estimated APS was then subtracted from the differential interferograms, and the regression-based estimation was iterated. After several iterations, the APS was well isolated, and we obtained iteratively refined estimates of the residual topography and linear deformation velocity.

In case of urban areas, particularly when sensing highrise buildings, the residual phase possibly contains a phase component associated with nonlinear deformation that must be differentiated from the atmospheric phase. We used another regression-based routine that models the nonlinear deformation as thermal expansion, assuming that the phase variations due to thermal expansion are linearly dependent on the temperature changes [40]. The regression coefficient is the phase-totemperature sensitivity, $\kappa$ [40], [43], [44]. The sensitivity is a measure of the phase variations undergone by a scatterer per unit change in temperature; the higher the sensitivity, the more pronounced is the thermal expansion. In this paper, we used a single temperature value for the entire scene, i.e., one temperature per acquisition, for each layer, optimized in a way that minimizes the residual phase in each layer [40].
Toward the end of the processing, the PSI solution obtained on the reduced candidate list was expanded to include all the candidates in the initial list, excluding those that failed to satisfy the quality criterion. For more details on various interferometric processing strategies using the IPTA toolbox, the interested readers are referred to earlier works [13], [40], [61]-[63]. Throughout the rest of this paper, the term "PSs identified with the PSI processing" refers to the final set of candidates from the last iteration.

\section{B. Tomographic Phase Models}

For a given range-azimuth pixel exhibiting ideal point scattering from one or more targets, assuming no additive or multiplicative noise, the mathematical model for classical SAR tomography (3-D SAR) can be written as [23], [27], [30], [43], [51]

$$
y_{n}=\int_{\triangle s} \gamma(s) \exp \left[-j \varphi_{n}(s)\right] d s
$$

$y_{n}$ is the SLC pixel value in $n$th layer of the coregistered and phase calibrated interferometric stack, for $n=0,1, \ldots, N-1$. $\gamma(s)$ is the target reflectivity profile along the elevation $s . \Delta s$ represents the observed elevation extent. In the case of 3-D SAR, the scatterer(s) is assumed stationary, and the interferometric phase is modeled as follows:

$$
\varphi_{n}(s)=2 k \triangle r_{n}(s)
$$

where $\triangle r_{n}(s)$ is the sensor-to-target path-length difference for the interferometric pair

$$
\triangle r_{n}(s)=r_{n}(s)-r_{0}(s) \approx \frac{s^{2}}{2\left(r_{0}-b_{n}^{\|}\right)}-\frac{b_{n}^{\perp} s}{r_{0}-b_{n}^{\|}} .
$$

The range distance from sensor $n$ to the scatterer at elevation $s$ is represented by $r_{n}$. The orthogonal and parallel components of the $n$th spatial baseline are $b_{n}^{\perp}$ and $b_{n}^{\|}$, respectively. The layer $n=0$ is considered the reference layer. The phase model for 3-D SAR depends only on the sensor-to-target geometry. It suffices in case the scatterer(s) remains stationary along the entire time series. However, in the case that there is some motion, there would be additional phase variations which must be accounted for in the phase model. Assuming that the motion is a temporally linear displacement in the LOS over the entire time series, differential tomography [31], [35], [38] extends the phase model as follows:

$$
\varphi_{n}(s, \nu)=2 k\left[\triangle r_{n}(s)+\nu t_{n}\right]
$$

where $\nu$ is the (average) linear deformation velocity of the scatterer, and $t_{n}$ is the temporal baseline for the $n$th layer. The SLC pixel value from (2) can now be recast (as shown in [26], [33], and [41]) as

$$
y_{n}=\iint_{\triangle s \triangle \nu} \gamma(s, \nu) \exp \left[-j \varphi_{n}(s, \nu)\right] d s d \nu
$$


where $\Delta \nu$ is the range of the expected linear deformation velocity. Different studies, such as [17], [19], [39], and [40], have shown that scatterers in urban areas may additionally be subject to nonlinear motion due to thermal expansion of the buildings. Assuming that the LOS phase change due to thermal expansion of building structures is linearly dependent on temperature changes, the tomographic phase model is further extended as follows:

$$
\varphi_{n}(s, \nu, \kappa)=2 k\left[\triangle r_{n}(s)+\nu t_{n}+\frac{1}{2 k} \kappa \tau_{n}\right]
$$

where $\kappa$ is the unknown phase-to-temperature sensitivity exhibited by the scatterer (or rather by the underlying physical structure) and $\tau_{n}$ is the temperature change (with respect to the temperature for the reference layer) for $n$th layer. With this phase model, the SLC pixel value is given by the following multivariate integral [45]:

$$
y_{n}=\iiint_{\triangle s \triangle \nu \triangle \kappa} \gamma(s, \nu, \kappa) \exp \left[-j \varphi_{n}(s, \nu, \kappa)\right] d s d \nu d \kappa .
$$

$\triangle \kappa$ is the range in which the phase-to-temperature sensitivity values of the scatterer(s) is expected. These values can be related to the linear expansion coefficients of the materials [40].

For brevity, we label the aforementioned phase models as follows: "P1" for the classical tomography (2), "P2" for differential tomography (4), and "P3" for the extended phase model, which simultaneously models thermal expansion besides scatterer elevation and linear deformation (6). A general mathematical model for SAR tomography can be defined as follows:

$$
y_{n}=\int_{\mathbb{P}} \gamma(\mathbf{p}) \exp \left[-j \varphi_{n}(\mathbf{p})\right] d \mathbf{p}
$$

where $\mathbf{p}$ is the unknown parameter vector for the scatterer and $\mathbb{P}$ represents the observed extent of the scatterer parameters, i.e., the parameter space. In the case of the phase model P1, $\mathbf{p}=[s]$; for P2, $\mathbf{p}=[s, \nu]$; and for P3, $\mathbf{p}=[s, \nu, \kappa]$.

\section{Single-Look Tomographic Inversion: Parameter Estimation and Scatterer Detection}

The estimation of the scatterer reflectivity $\gamma(\mathbf{p})$ and the scatterer parameter vector $\mathbf{p}$ is an inverse problem, i.e., the tomographic model in (8) has to be inverted starting with the SLC values (measurements). Additionally, a detection strategy has to be applied to ascertain whether a given pixel contains any coherent scatterers.

In urban areas, layover scenarios occur frequently. A layoveraffected pixel may contain backscatter contribution from two or more scatterers that may individually be temporally coherent. In this paper, considering that a given pixel has a maximum of two coherent scatterers, we make the following hypotheses: $\mathcal{H}^{0}$ - the pixel does not represent a stable scatterer, i.e., the SLC values represent merely noise; $\mathcal{H}^{1}-$ the pixel is a single scatterer; or $\mathcal{H}^{2}$ - the pixel is a double scatterer. The hypotheses are defined mathematically as follows [38]:

$$
\begin{aligned}
& \mathcal{H}^{0}: \mathbf{y}=\mathbf{n} \\
& \mathcal{H}^{1}: \mathbf{y}=\gamma_{1} \mathbf{a}\left(\mathbf{p}_{1}\right)+\mathbf{n} \\
& \mathcal{H}^{2}: \mathbf{y}=\gamma_{1} \mathbf{a}\left(\mathbf{p}_{1}\right)+\gamma_{2} \mathbf{a}\left(\mathbf{p}_{2}\right)+\mathbf{n} .
\end{aligned}
$$

$\mathbf{y}$ is the SLC vector, i.e.,

$$
\mathbf{y}=\left[\begin{array}{llll}
y_{0} & y_{1} & \cdots & y_{N-1}
\end{array}\right]^{T} .
$$

$\gamma$ represents scatterer reflectivity, and $\mathbf{a}(\mathbf{p})$ is the steering vector; the subscripts 1 and 2 indicate the first and the second scatterer in the pixel, respectively, with the first having more energy compared with the second. The general structure of the steering vector as a function of the unknown parameter vector $p$ is given by

$$
\mathbf{a}(\mathbf{p})=\left[\begin{array}{llll}
1 & e^{-j \varphi_{1}(\mathbf{p})} & \cdots & e^{-j \varphi_{N-1}(\mathbf{p})}
\end{array}\right]^{T} .
$$

$\mathbf{n}$ is the random noise vector generally considered zero-mean circular Gaussian. The structure of the phase values $\varphi_{n}(\mathbf{p})$, and the unknowns in the parameter vector $\mathbf{p}$, depend on the choice of the phase model [(2), (4), and (6)].

Different methods have been proposed over time for tomographic inversion and scatterer detection, as in [26], [30], [34], [38], and [51]-[54]. In this paper, we use the reflectivity of single-look BF as a merit function for the parameter estimation problem. It can be directly associated with GLRT-based scatterer detection strategies [34], [38]. The reflectivity estimated with $\mathrm{BF}$ as a function of the parameter vector, $\mathbf{p}$ is given by

$$
\hat{\gamma}(\mathbf{p})=\frac{1}{N} \mathbf{a}^{H}(\mathbf{p}) \mathbf{y} .
$$

$\mathrm{BF}$ is a nonparametric method. The presence of one or more scatterers manifests in the peaks of the estimated absolute or squared reflectivity. Assuming the presence of at least one scatterer (i.e., either hypothesis $\mathcal{H}^{1}$ or $\mathcal{H}^{2}$ is true), we use the estimated absolute reflectivity as the objective function in the following optimization:

$$
\hat{\mathbf{p}}_{1}=\underset{\mathbf{p} \in \mathbb{P}}{\arg \max }(|\hat{\gamma}(\mathbf{p})|) .
$$

The parameters in $\mathbb{P}$ that globally maximize the merit function are the estimated parameters for the first scatterer $\hat{\mathbf{p}}_{1}$. It is implicitly assumed that the steering vector for the first scatterer is orthogonal to both the noise vector and the steering vector of a second scatterer, if any. The estimated energy of the first scatterer $\hat{E}_{1}$ (normalized with the total energy of the SLC vector) is as follows:

$$
\hat{E}_{1}=\frac{\left|\hat{\gamma}\left(\hat{\mathbf{p}}_{1}\right)\right|^{2}}{\|\mathbf{y}\|^{2}}
$$

In the case that a second scatterer exists in the same pixel, i.e., hypothesis $\mathcal{H}^{2}$ is true; in principle, we may look for a second peak of the estimated reflectivity. However, a sidelobe of the first scatterer can always be mistaken for a second scatterer and thus lead to false detection. An alternative way suggested by 
[38] is to subtract the estimated backscatter of the first scatterer from the SLC vector and search for a potential second scatterer by applying inversion on the difference $\mathbf{y}_{c}$, i.e.,

$$
\begin{aligned}
\mathbf{y}_{c} & =\mathbf{y}-\hat{\gamma}\left(\hat{\mathbf{p}}_{1}\right) \mathbf{a}\left(\hat{\mathbf{p}}_{1}\right) \\
& =\hat{\mathbf{P}}_{1}^{\perp} \mathbf{y}
\end{aligned}
$$

where

$$
\hat{\mathbf{P}}_{1}^{\perp}=\mathbf{I}_{N}-\frac{\mathbf{a}\left(\hat{\mathbf{p}}_{1}\right) \mathbf{a}^{H}\left(\hat{\mathbf{p}}_{1}\right)}{N} .
$$

$\mathbf{I}_{N}$ is the identity matrix of size $N . \hat{\mathbf{P}}_{1}^{\perp}$ can be considered the projector onto the orthogonal complement of the subspace spanned by the steering vector of the first scatterer $\mathbf{a}^{H}\left(\hat{\mathbf{p}}_{1}\right)$. The parameters for a potential second scatterer, $\hat{\mathbf{p}}_{2}$ are computed by applying the following BF-based maximization [38]:

$$
\hat{\mathbf{p}}_{2}=\underset{\mathbf{p} \in \mathbb{P}}{\arg \max }\left(\frac{\left|\mathbf{a}^{H}(\mathbf{p}) \mathbf{y}_{c}\right|}{\left\|\hat{\mathbf{P}}_{1}^{\perp} \mathbf{a}(\mathbf{p})\right\|}\right) .
$$

The estimated energy of the second scatterer $\hat{E}_{2, c}$, obtained after canceling the first scatterer from the SLC vector and normalized with the energy of the difference $\mathbf{y}_{c}$, is given by

$$
\hat{E}_{2, c}=\frac{\left|\mathbf{u}_{c}^{H} \mathbf{y}_{c}\right|^{2}}{\left\|\mathbf{y}_{c}\right\|^{2}}
$$

where

$$
\mathbf{u}_{c}=\frac{\hat{\mathbf{P}}_{1}^{\perp} \mathbf{a}\left(\hat{\mathbf{p}}_{2}\right)}{\left\|\hat{\mathbf{P}}_{1}^{\perp} \mathbf{a}\left(\hat{\mathbf{p}}_{2}\right)\right\|} .
$$

To distinguish between the three hypotheses $\left(\mathcal{H}_{0}, \mathcal{H}_{1}\right.$, and $\mathcal{H}_{2}$ ), we use the sequential generalized likelihood ratio test with cancellation (SGLRTC), as proposed in [38]. SGLRTC compares the normalized energy of the scatterers, $\hat{E}_{1}$ and $\hat{E}_{2, c}$, against preselected thresholds, $T_{1}$ and $T_{2}$, respectively. The test is sequential, as it comprises of two consecutive steps. First, it is decided whether the pixel is a double scatterer or not, i.e., $\mathcal{H}_{2}$ or $\overline{\mathcal{H}}_{2}$, respectively, i.e.,

$$
\hat{E}_{2, c} \underset{\mathcal{H}_{2}}{\stackrel{\mathcal{H}_{2}}{\gtrless}} T_{2} .
$$

If it is decided that the pixel is not a double scatterer, then it is tested against hypothesis $\mathcal{H}_{1}$ (the pixel contains a single scatterer) and $\mathcal{H}_{0}$, (no scatterer detected), respectively, i.e.,

$$
\hat{E}_{1} \underset{\mathcal{H}_{0}}{\stackrel{\mathcal{H}_{1}}{\gtrless}} T_{1} \text {. }
$$

The lower the thresholds, the more relaxed are the requirements on the minimum normalized energy to be detected as coherent scatterers. If the thresholds are too low, the energy of the clutter/noise may exceed the thresholds leading to false alarms. Similarly, a very high threshold may lead to missed detection. The choice of the thresholds can be associated with the desired probabilities of detection and false alarm. As suggested in [38], we keep the thresholds equal $\left(T_{1}=T_{2}\right)$ to jointly maximize the probabilities of detection for both the single and double scatterers for a given probability of false alarm. For further details, interested readers are referred to [38] for a comprehensive discussion and comparison of SGLRTC with other detection strategies.

Although the thresholds $T_{1}$ and $T_{2}$ can be set to attain the desired probabilities of detection and false alarm, it remains to be assessed whether the thresholds can be related to the quality of the parameter estimates. We consider that the quality of the estimates is represented by the goodness of fit of the tomographic model with actual measurements (SLC values). Using the estimated parameters, we compute the estimated SLC vector $\mathbf{y}_{\text {est }}$ (model fit) and observe phase deviation between the estimated and the actual SLC values in each layer. Low phase deviations represent a better fit of the tomographic model to the measurements. We compute the RMS phase deviation as a metric for the goodness of fit and hence as a metric for the quality of the estimates. In mathematical terms, the estimated SLC vector $\mathbf{y}_{\text {est }}$ for the single and double scatterers detected with SGLRTC is given by

$$
\mathbf{y}_{\text {est }}= \begin{cases}\hat{\gamma}_{1} \mathbf{a}\left(\hat{\mathbf{p}}_{1}\right) & \text { single scatterer } \\ \hat{\gamma}_{1} \mathbf{a}\left(\hat{\mathbf{p}}_{1}\right)+\hat{\gamma}_{2} \mathbf{a}\left(\hat{\mathbf{p}}_{2}\right) & \text { double scatterer }\end{cases}
$$

where $\hat{\gamma}_{1}$ and $\hat{\gamma}_{2}$ are the estimated reflectivities of the first and second scatterer, respectively:

$$
\begin{aligned}
& \hat{\gamma}_{1}=\mathbf{a}^{H}\left(\hat{\mathbf{p}}_{1}\right) \mathbf{y} \\
& \hat{\gamma}_{2}=\mathbf{a}^{H}\left(\hat{\mathbf{p}}_{2}\right) \mathbf{y}_{c} /\left\|\hat{\mathbf{P}}_{1}^{\perp} \mathbf{a}\left(\hat{\mathbf{p}}_{2}\right)\right\| .
\end{aligned}
$$

The phase deviation for the $n$th layer, $\varphi_{n}^{\text {res }}$ is computed as follows:

$$
\varphi_{n}^{\text {res }}=\mathrm{AD}\left\{\measuredangle y_{n}, \measuredangle y_{n, \text { est }}\right\}
$$

where $y_{n \text {,est }}$ is the $n$th element of the vector $\mathbf{y}_{\text {est }}, \measuredangle(\cdot)$ returns the phase of the complex argument between 0 and $2 \pi$, and the operator $\operatorname{AD}\{.,$.$\} returns the absolute angle deviation between$ its arguments as follows:

$$
\mathrm{AD}\left\{w_{1}, w_{2}\right\}= \begin{cases}d & d \leq \pi \\ 2 \pi-d & d>\pi\end{cases}
$$

for $d=\left|w_{1}-w_{2}\right|$. The RMS phase deviation, $\sigma^{\text {tomo }}$ is given by

$$
\sigma^{\text {tomo }}=\sqrt{\frac{1}{N-1} \sum_{n=1}^{N}\left(\varphi_{n}^{\text {res }}\right)^{2}} .
$$

The quality of the estimates can also be related to the energy of the residue, i.e., the squared norm of the model mismatch $\left\|\mathbf{y}-\mathbf{y}_{\text {est }}\right\|^{2}$. Low residual energy would imply a better match. However, in this paper, we use phase deviations as a measure of model mismatch in order to be consistent with the quality assessment in IPTA-based PSI processing (which ignores amplitudes and operates only on the phases of the complex SLC values in the regression-based parameter estimation). 


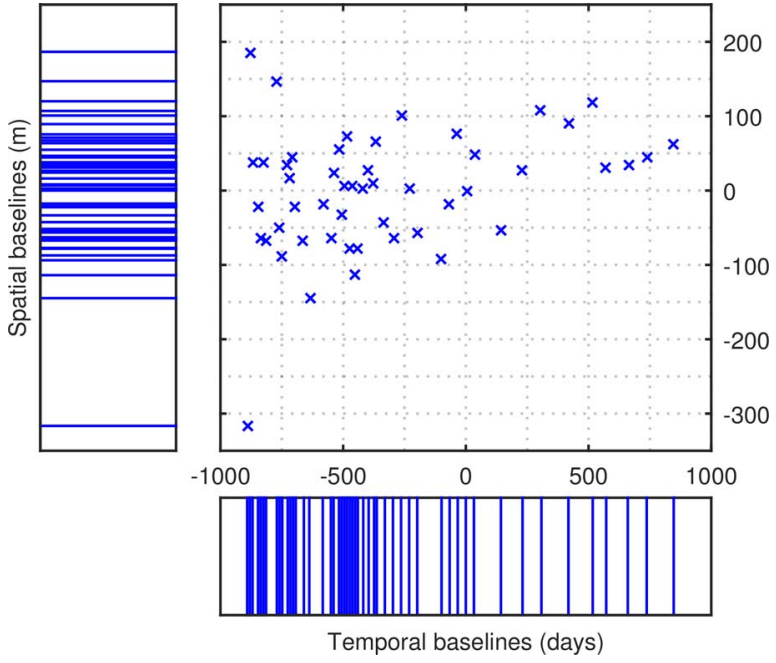

(a)

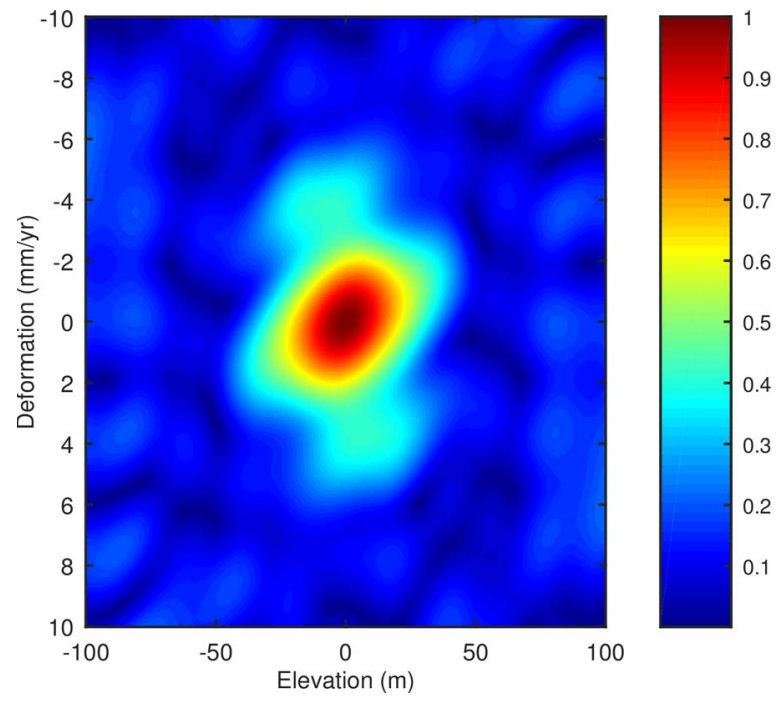

(b)

Fig. 1. Characteristics of the interferometric data stack used in this paper. (a) Distribution of the temporal baselines and the spatial baselines (orthogonal component). (b) Two-dimensional point spread function in elevation-deformation plane.

\section{DATA STACK}

The interferometric data stack used in this paper comprises 50 TerraSAR-X stripmap images acquired in repeated passes over the city of Barcelona, Spain. The images are spread over a time span of approximately 5 years, from December 2007 to October 2012. The polarization mode of the images is VV. The slant range resolution $\delta_{r}$ is $1.2 \mathrm{~m}$, corresponding to a chirp bandwidth of $150 \mathrm{MHz}$. The resolution in azimuth is $3.3 \mathrm{~m}$. The images were oversampled by a factor of 2 to allow for a more accurate coregistration. The pixel spacing in range and azimuth is 0.455 and $1.89 \mathrm{~m}$, respectively.

The distribution of both the temporal and spatial baselines is highly nonuniform, as shown in Fig. 1(a). The total orthogonal spatial baseline i.e., the aperture in elevation $B^{\perp}$, is $503.2 \mathrm{~m}$. The resolution in elevation $\delta_{s}$, had the spatial baselines been uniformly distributed, would be

$$
\delta_{s}=\frac{\lambda r_{0}}{2 B^{\perp}}=19.2 \mathrm{~m}
$$

where $\lambda=3.1 \mathrm{~cm}$ and $r_{0}=622.8 \mathrm{~km}$. The corresponding resolution in height would be $11.1 \mathrm{~m}$, for an incidence angle of $35.3^{\circ}$ for the reference layer. The resolution in deformation velocity, assuming uniform temporal baselines, is

$$
\delta_{\nu}=\frac{\lambda}{2 T}=3.26 \mathrm{~mm} \cdot \mathrm{yr}^{-1}
$$

where $T=1738$ days is the total temporal span. Due to the nonuniformity of the baselines, the point spread function (PSF) is distorted, as shown in Fig. 1(b). For a given range bin, the range migration due to the different viewing angles in each pass should be very small compared with the range resolution range, which sets an upper limit on the observed extent of elevation $\triangle s[30]$

$$
\triangle s \ll \frac{\delta_{r} r_{0}}{B^{\perp}}=1485 \mathrm{~m} .
$$

Although we would expect an ambiguity in tomographic inversion with uniform baselines at an elevation of $942 \mathrm{~m}$, the randomness in the distribution of the real baselines proves useful in alleviating the ambiguity over the entire observed extent.

\section{REsults}

This section presents the results obtained on the interferometric data stack introduced earlier. The urban area under study is Diagonal Mar, Barcelona, Spain. The average SAR backscatter image and the corresponding Google image of the area are shown in Fig. 2. There are various man-made structures in the scene which may exhibit temporally coherent scattering. The area includes both layover (high-rise buildings) and nonlayover affected areas; therefore, it exemplifies a general urban scenario from an operational point of view. As the temporal span of the acquisitions in the data stack extends over many seasons, we expect to see some nonlinear deformation (besides any possible linear deformation) due to the thermal expansion of the building structures.

\section{A. PSI Solution}

We perform a PSI analysis of the data stack using the IPTA [13] framework. The final solution is obtained after several iterations of the phase regressions for the estimation of scatterer residual height, linear deformation and the phaseto-temperature sensitivity, and the separation of the APS from the interferometric stack. In this paper, we used a threshold of $1.1 \mathrm{rad}$ for the RMS phase deviation $\sigma^{\text {ipta }}$ i.e., the set of PSCs with $\sigma^{\text {ipta }}$ greater than $1.1 \mathrm{rad}$ were rejected in the iterative regression-based parameter estimation. Fig. 3 shows the PSs identified in the PSI processing and the estimated scatterer parameters (relative height, LOS deformation velocity, and phase-to-temperature sensitivity). 

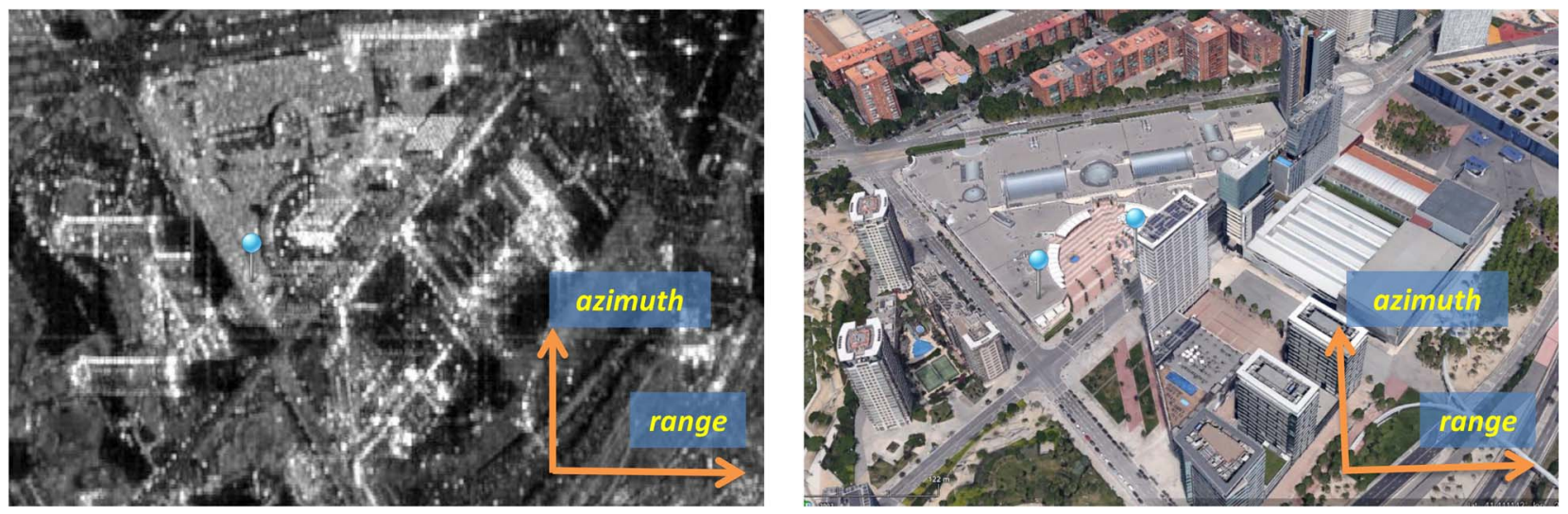

Fig. 2. Left: TerraSAR-X averaged intensity image of the observed urban area (Diagonal Mar, Barcelona, Spain). Right: Google Earth snapshot of the area. The blue pins mark the location of an example layover-affected pixel (a double scatterer containing signal returns from both the facade of the high-rise building as well as the rooftop of the triangular Diagonal Mar shopping center).

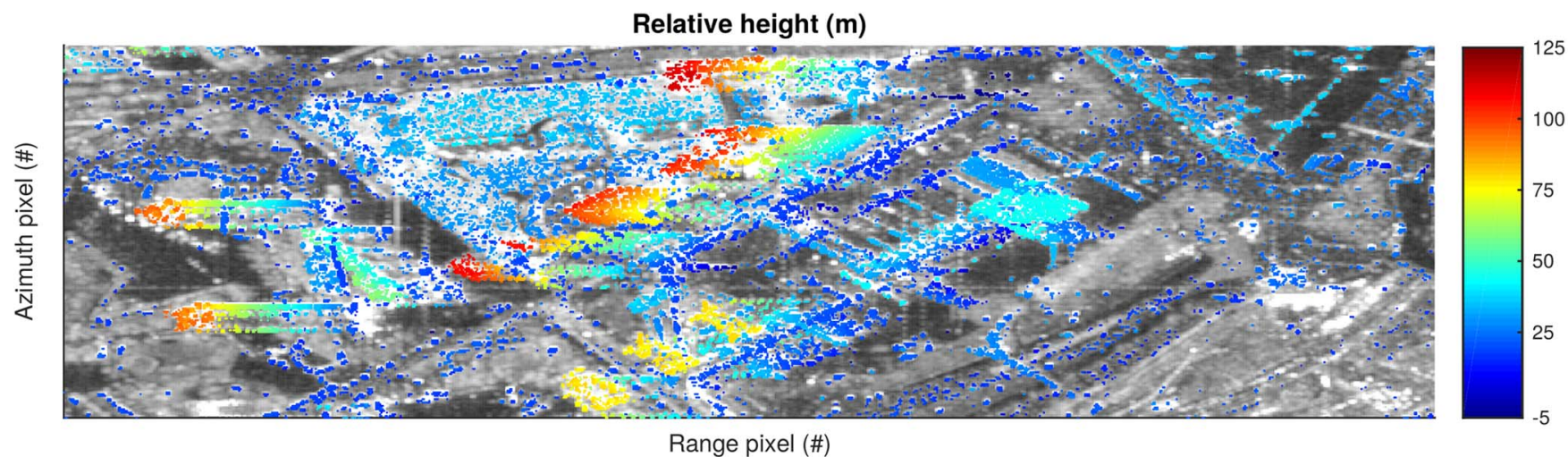

\section{LOS Deformation velocity (mm/yr)}

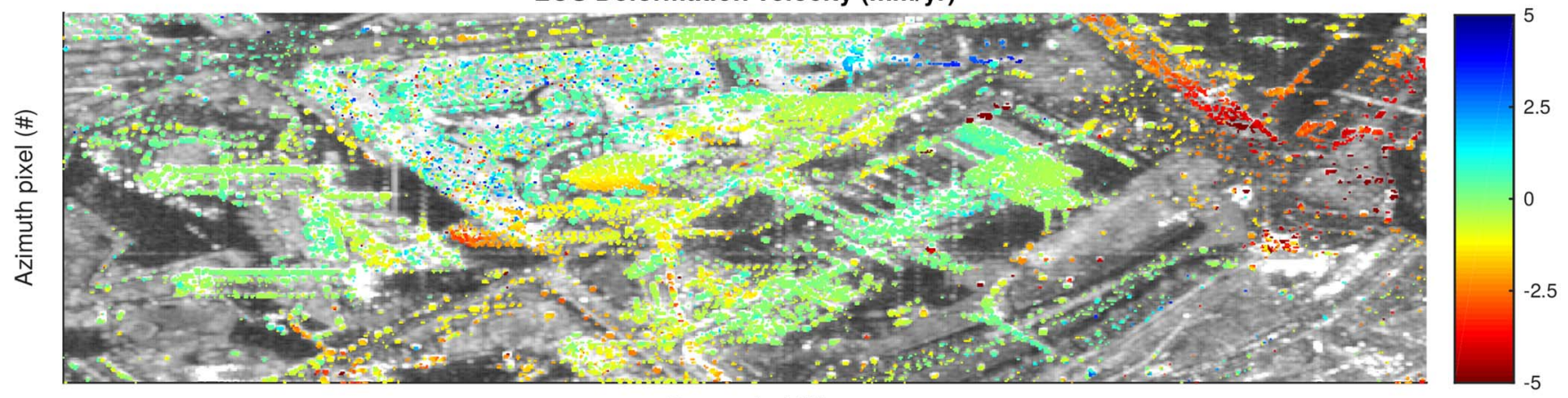

Range pixel (\#)

Phase-to-temperature sensitivity ( $\mathrm{rad} / \mathrm{K})$

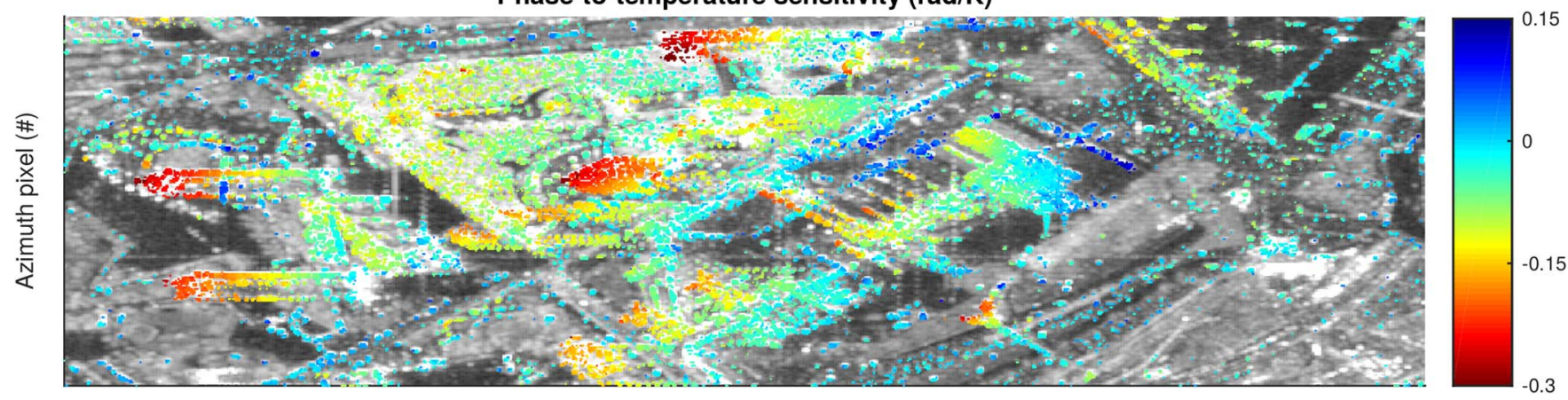

Range pixel (\#)

Fig. 3. PSI solution obtained with the IPTA framework. The colored dots are the PSs identified in the PSI processing. (Top) Estimated height, relative to WGS-84 reference ellipsoid. Middle: Deformation velocity in the line-of-sight. (Bottom) Phase-to-temperature sensitivity. 


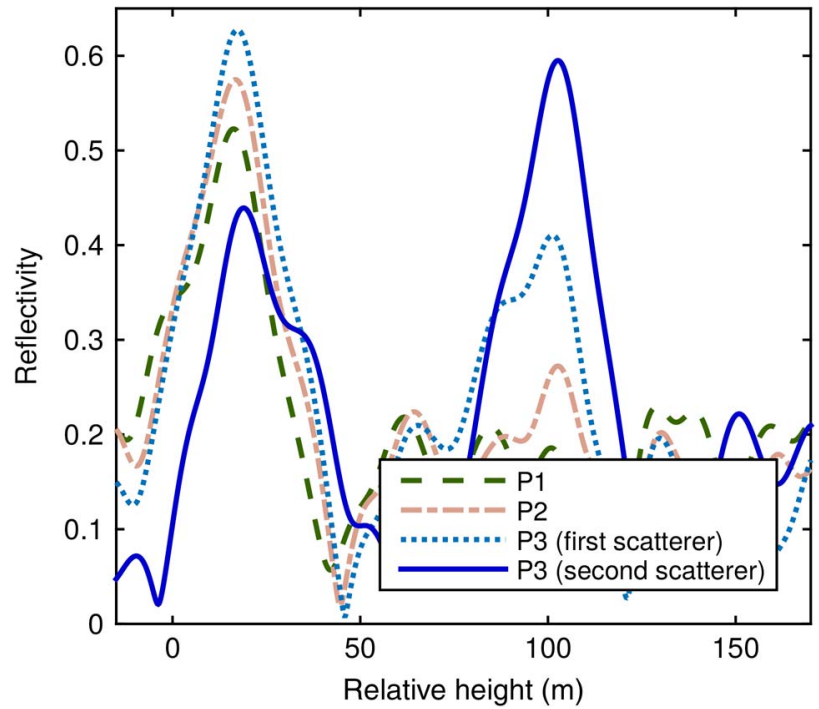

Fig. 4. Reflectivity profiles obtained with tomography for an example layoveraffected pixel [as marked with blue pins in Fig. 2]. Results are shown for three different phase models: P1 (3-D SAR), P2 (differential tomography), and P3 (elevation, linear deformation, and thermal expansion are simultaneously modeled). The elevation coordinate has been projected to vertical (relative) height.

\section{B. Tomographic Inversions and Scatterer Detection}

Prior to tomographic inversion, the interferometric stack needs to be phase calibrated. The APS isolated in the PSI processing for the PSs is extrapolated to all the pixels in the scene, and subtracted from the differential interferograms to allow for single-look tomographic inversion on all pixels. The reference layer, spatial and temporal baselines, and the reference point used for the tomographic inversion are the same as in the PSI processing. The reference temperature values used for modeling thermal expansion are as refined in the PSI processing (such that the residual phase in each layer is minimized [40]). The estimation of scatterer parameters (elevation, deformation velocity, and phase-to-temperature sensitivity) with BF-based tomographic inversion is otherwise independent of the PSI solution. The observed extents of the parameters are as follows: $\triangle s=[-50,300] \mathrm{m}, \triangle \nu=[-5,5] \mathrm{mm} / \mathrm{yr}$ and $\triangle \kappa=[-1,1] \mathrm{rad} / \mathrm{K}$.

1) Layover-Affected Pixel: The blue pins in Fig. 2 mark the location of a layover-affected pixel which has not been identified as a PS in the PSI processing. It is expected that the pixel contains two scatterers: one situated on the facade of a tall building, whereas the other on the rooftop of a nearby building of relatively short height. We apply tomographic inversion for this pixel with each of the three different phase models discussed in Section II-B. The reflectivity profiles retrieved for each phase model are shown in Fig. 4. For the case of classical SAR tomography [phase model P1, (2)], the reflectivity profile shows the presence of a single scatterer around $20 \mathrm{~m}$. Similarly, differential tomography [phase model P2, (4)] also reveals the presence of a single scatterer. In addition to height, differential tomography models temporally linear deformation. Fig. 5 shows the 2-D reflectivity in the height-deformation plane.

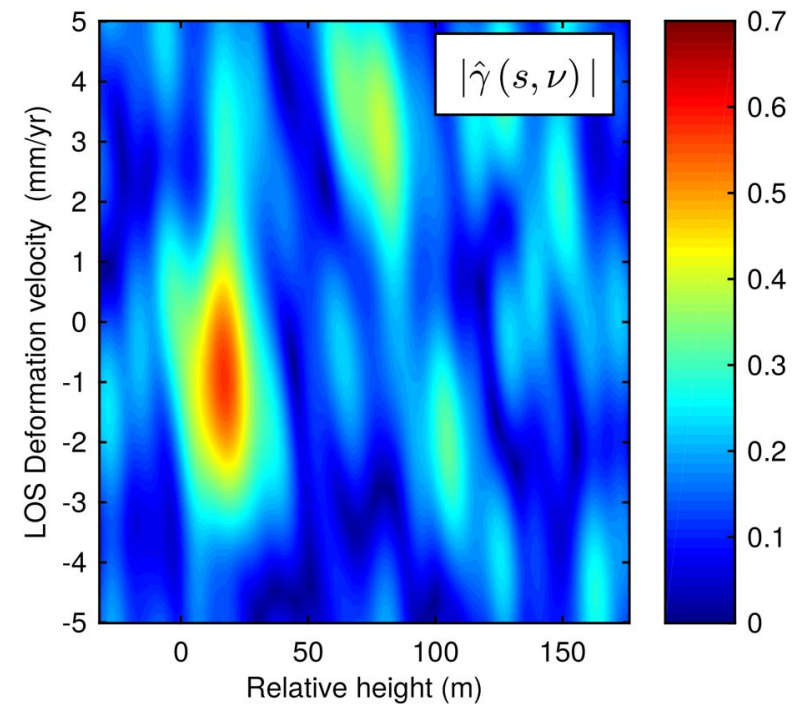

Fig. 5. Reflectivity in the height-deformation plane obtained with phase model P2 (differential tomography), i.e., $|\hat{\gamma}(\mathbf{p})|$ with $\mathbf{p}=[s, \nu]$ [see (4)].

Tomographic inversion with the extended phase model [which simultaneously models scatterer height, linear deformation, and thermal expansion; P3, (6)], is also applied. The 3-D reflectivity space is shown in Fig. 6. It can now be seen that the pixel is in fact a double scatterer, containing two individual scatterers of nearly comparable energy. The layover is resolved. The estimated heights of the first and the second scatterers are nearly 20 and $100 \mathrm{~m}$, respectively. The estimated normalized energy for the first scatterer is 0.6 , whereas the normalized energy estimated for the second scatterer (after the cancelation of the first with SGLRTC) is 0.4 .

2) Diagonal Mar: Tomographic inversion with the extended phase model P3 is applied on the entire Diagonal Mar area, for all the pixels, whether identified as PS or not during the PSI processing. Single and double scatterers are detected using the SGLRTC-based detection strategy. The number of scatterers detected with SGLRTC is directly influenced by the choice of the thresholds $T_{1}$ and $T_{2}$. Fig. 7 shows the variation in the number of the detected scatterers against the thresholds (while keeping $T_{1}=T_{2}$ ). The lower the thresholds, the more are the detected scatterers. However, for thresholds below 0.3, we observe a sudden jump in the number of double scatterers accompanied by a sharp decline in the gradient for single scatterers. For thresholds between 0.3-0.4, we have nearly twice as many scatterers obtained with tomography than the PSs identified with PSI. For thresholds greater than 0.6, only a very few double scatterers are detected and the total number of scatterers obtained with tomography drops below the number of PSs. At a moderate value of 0.4 for the thresholds, Fig. 8 shows the single scatterers, as well as the scatterers with higher elevation among the double scatterers (usually referred to as the upper layer), in radar coordinates. Figs. 9 and 10 show the single and double scatterers, respectively, projected on Google Earth 3-D building models. The color coding represents the estimated parameters. 


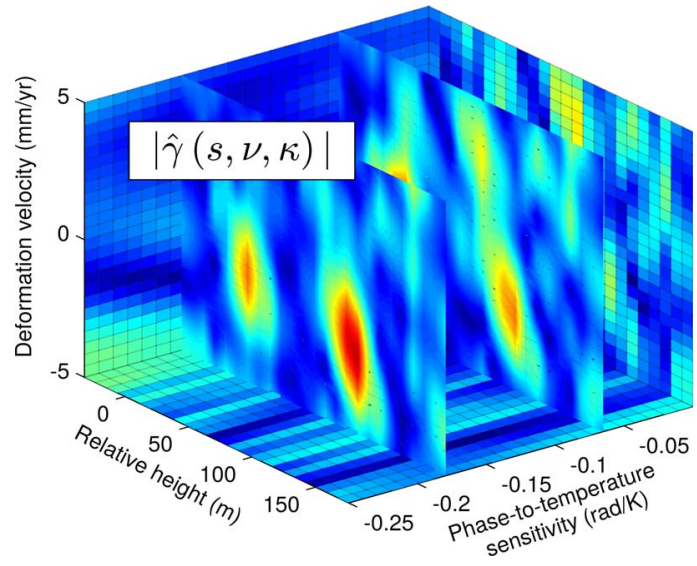

(a)

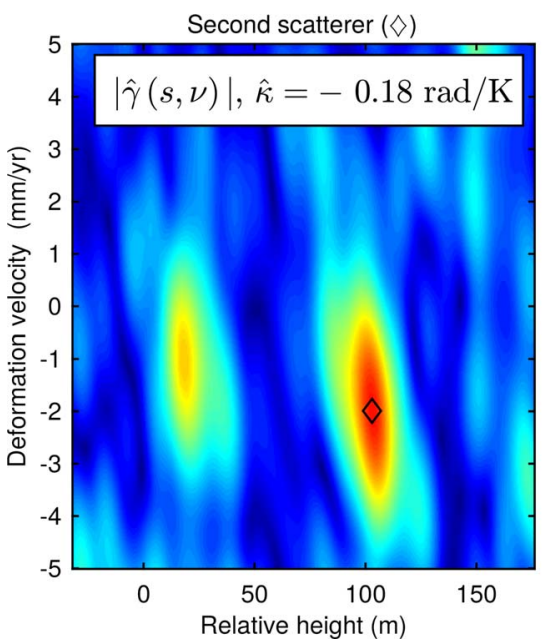

(c)

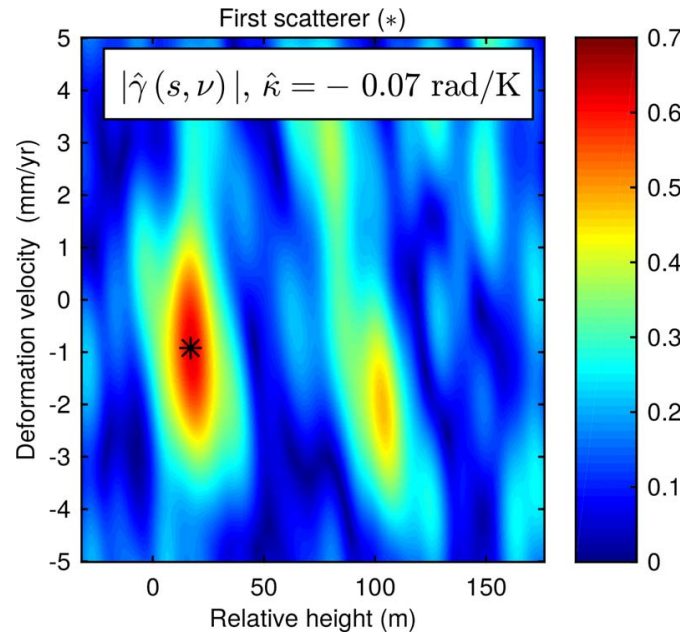

(b)

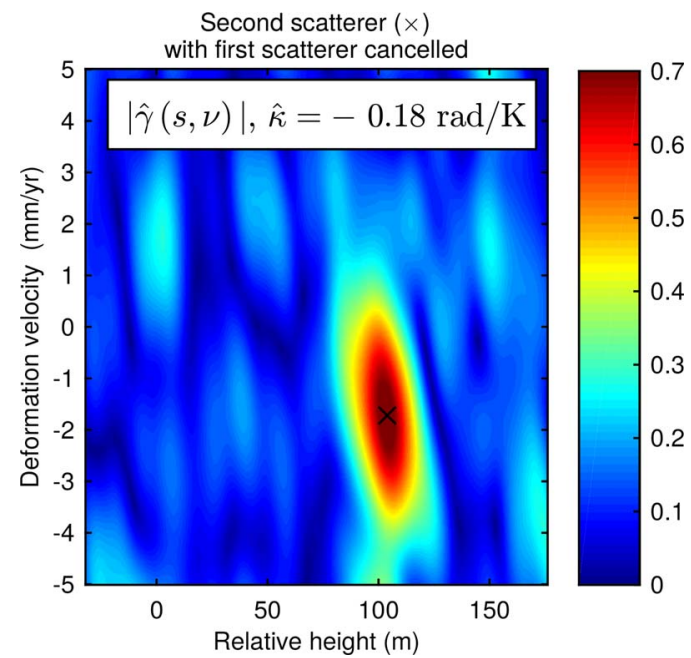

(d)

Fig. 6. Example of a tomographic inversion for the layover-affected pixel (as marked in Fig. 2 with blue pins) using the phase model P3, i.e., the scatterer elevation, temporally linear deformation and thermal expansion are modeled simultaneously [see (6)]. (a) Estimated 3-D reflectivity $|\hat{\gamma}(s, \nu, \kappa)|$. (b) and (c) Slices

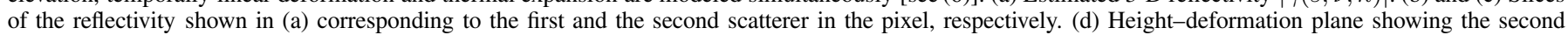
scatterer after the cancelation of the first using the SGLRTC [38].

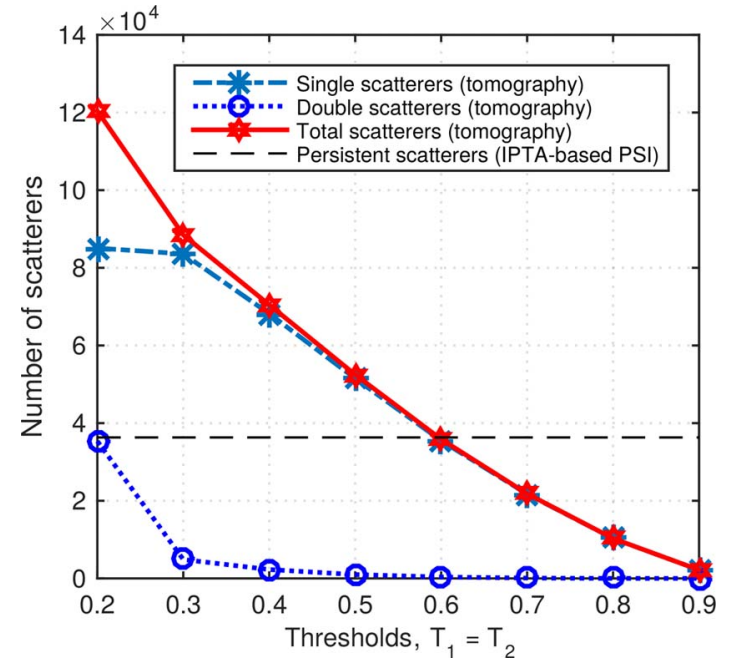

Fig. 7. Number of single and double scatterers detected in the Diagonal Mar area using tomographic inversion and SGLRTC [38], for different thresholds of detection $T_{1}$ and $T_{2}$, keeping $T_{1}=T_{2}$. The tomographic phase model used simultaneously models scatterer elevation, linear deformation, and thermal expansion [i.e., P3 (6)]. The number of PSs identified with the IPTA-based PSI processing are also given for reference.

\section{Relative Gain in Deformation Sampling With Layover Separations}

We intend to assess how effective is SAR tomography in increasing deformation sampling (by resolving layovers) in comparison to a PSI-based analysis. To this end, we define the relative gain in deformation sampling, $G$ as follows:

$$
G=\left(\frac{2 N_{d, u}+N_{d, \mathrm{psi}}}{N_{\mathrm{psi}}}\right) \times 100 \% .
$$

$N_{d, u}$ is the number of pixels that are uniquely detected as double scatterers, i.e., the pixels were not identified as PSs in the PSI processing; $N_{d, \mathrm{psi}}$ is the number of those pixels that are detected as double scatterers but were also identified as PSs; and $N_{\mathrm{psi}}=36312$ is the total number of the PSs identified in the scene. We would expect that a PSI analysis should reject all the pixels containing double scatterers. However, if one of the two scatterers tends to dominate the other, the pixel may still get selected as a PS. Fig. 11 shows the double scatterers and the corresponding gain at the different thresholds of detection. It can be seen that the relative gain drops sharply with increasing 


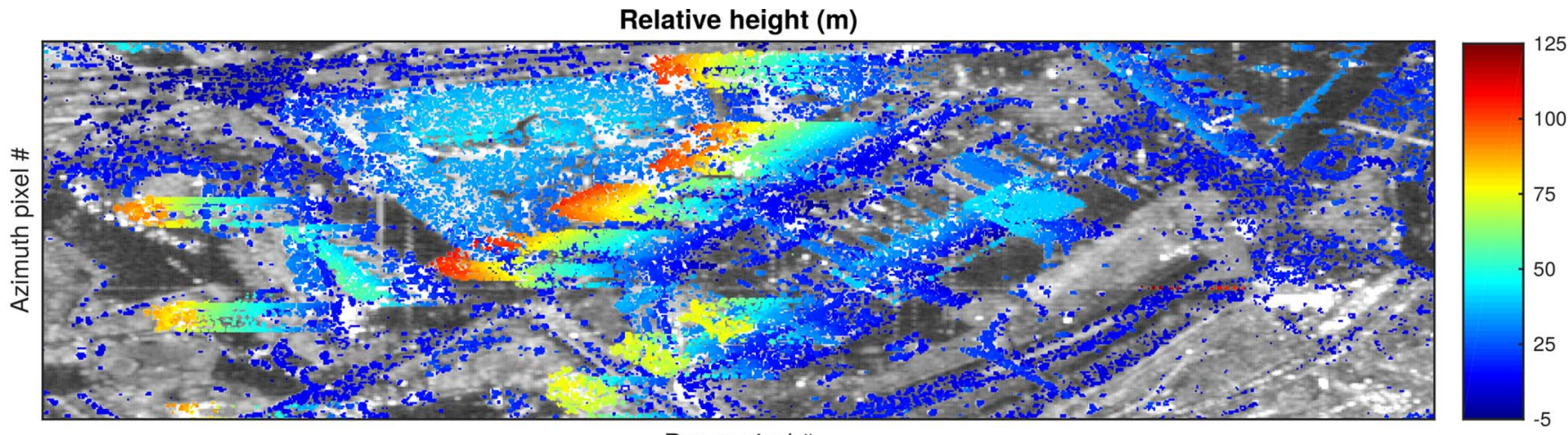

Range pixel \#

LOS Deformation velocity $(\mathrm{mm} / \mathrm{yr})$

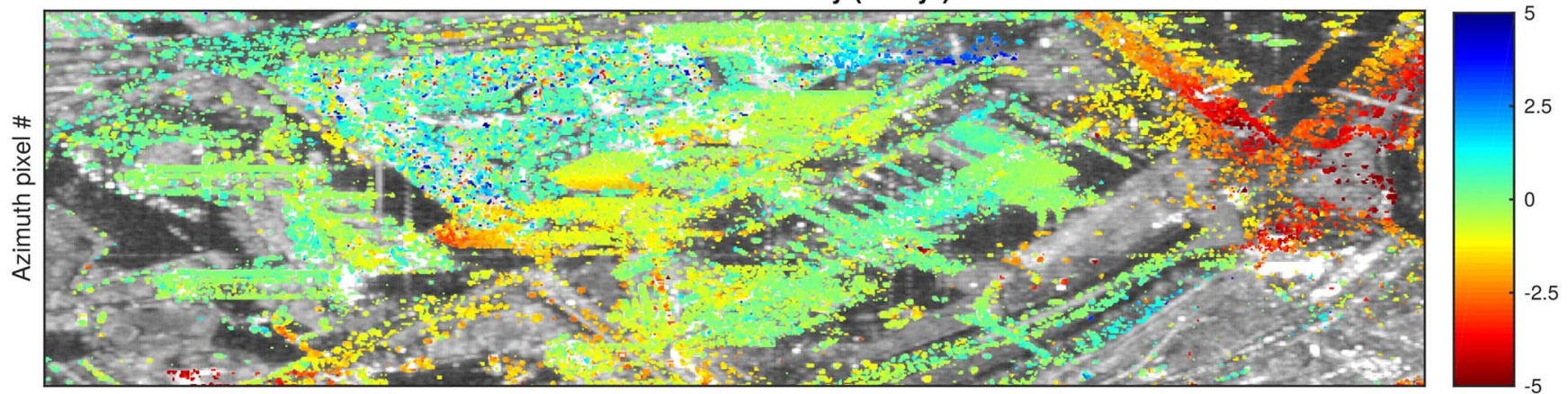

Range pixel \#

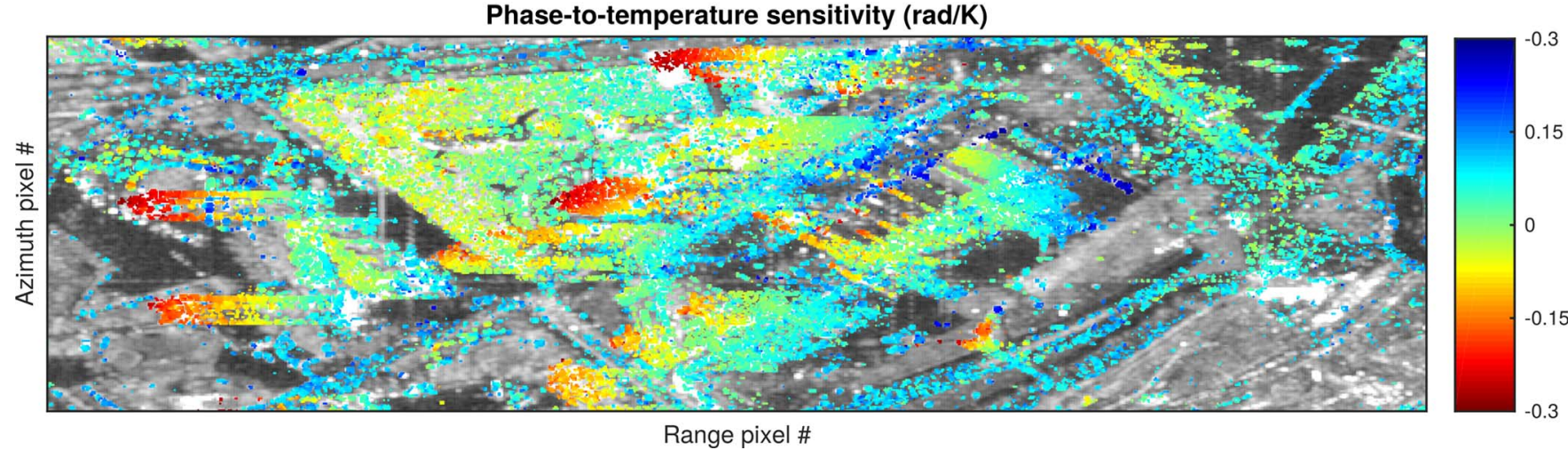

Fig. 8. Single scatterers alongside upper layer double scatterers obtained with tomographic inversion and SGLRTC. Thresholds for detection $T_{1}$ and $T_{2}$ are set at 0.4. The colors represent the estimated parameters. Top row: Relative height. Middle row: Average deformation velocity in LOS. Bottom row: Phase-to-temperature sensitivity.

thresholds. The choice of the thresholds should consider both the gain and the quality of the scatterers, as argued in the following subsection.

\section{Quality of the Estimates}

The quality of the estimates obtained with IPTA-based PSI processing and BF-based tomographic inversion is assessed in terms of the RMS phase deviations, $\sigma^{\text {ipta }}$ and $\sigma^{\text {tomo }}$, respectively. The lower the RMS phase deviation, the better is the perceived quality of the estimates.

1) PSs Identified With IPTA-Based PSI Processing: Fig. 12 shows the joint and marginal distributions of the $\sigma^{\text {tomo }}$ and $\sigma^{\text {ipta }}$ for the pixels that have been identified as the PSs in IPTA-based PSI processing. A strong correlation is clearly visible. The average values of the marginal distributions for tomography and PSI are 0.56 and $0.57 \mathrm{rad}$, respectively. There are a very few pixels $(<0.01 \%)$ for which $\sigma^{\text {tomo }}$ is higher than $1.1 \mathrm{rad}$, which was used as the upper limit in the IPTA-based PSI processing.

2) Single Scatterers Obtained With Tomography: Fig. 13(a) shows the distributions of the RMS phase deviation, $\sigma^{\text {tomo }}$ for the single scatterers obtained with tomography at different thresholds. It can be seen that progressively increasing the thresholds improves the overall quality, but alongside, there is a drop in the number of the detected scatterers.

Fig. 13(b) shows the distribution of RMS phase deviation, $\sigma^{\text {tomo }}$ for the single scatterers obtained with tomography in addition to the PSs identified with the PSI processing at various thresholds. The quality improves with increasing thresholds, but it can be seen that, on average, these scatterers exhibit a lower quality than for the PSs. To ensure that at least $95 \%$ of these pixels should have $\sigma^{\text {tomo }}$ below $1.1 \mathrm{rad}$, we need thresholds of 0.40 or higher. 


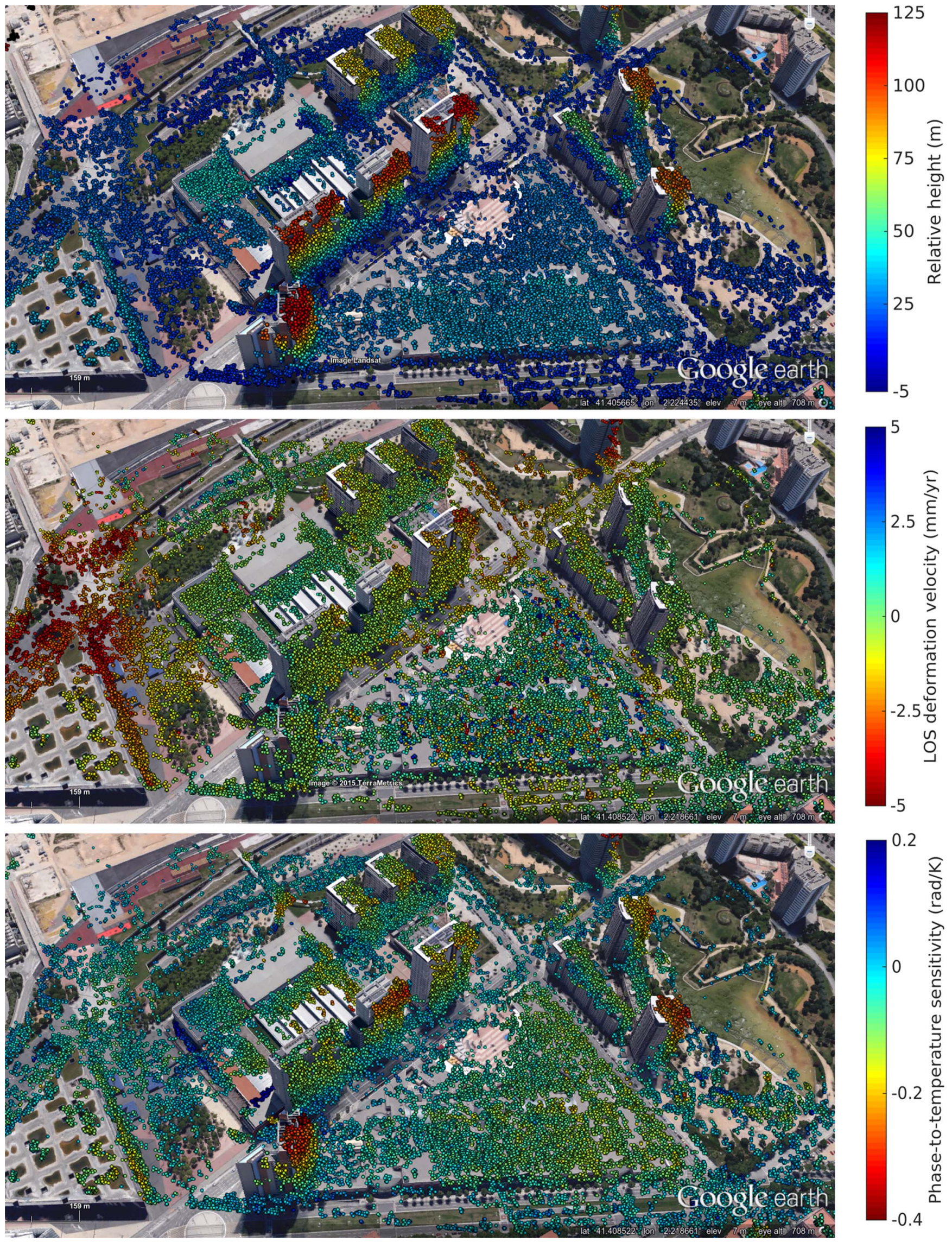

Fig. 9. Single scatterers obtained with tomographic inversion and SGLRTC, and projected on Google Earth 3-D building models. The thresholds for detection $T_{1}$ and $T_{2}$ are set at 0.4 . The colors represent the estimated parameters. (Top row) Relative height. (Middle row) Average deformation velocity in LOS. (Bottom row) Phase-to-temperature sensitivity. 


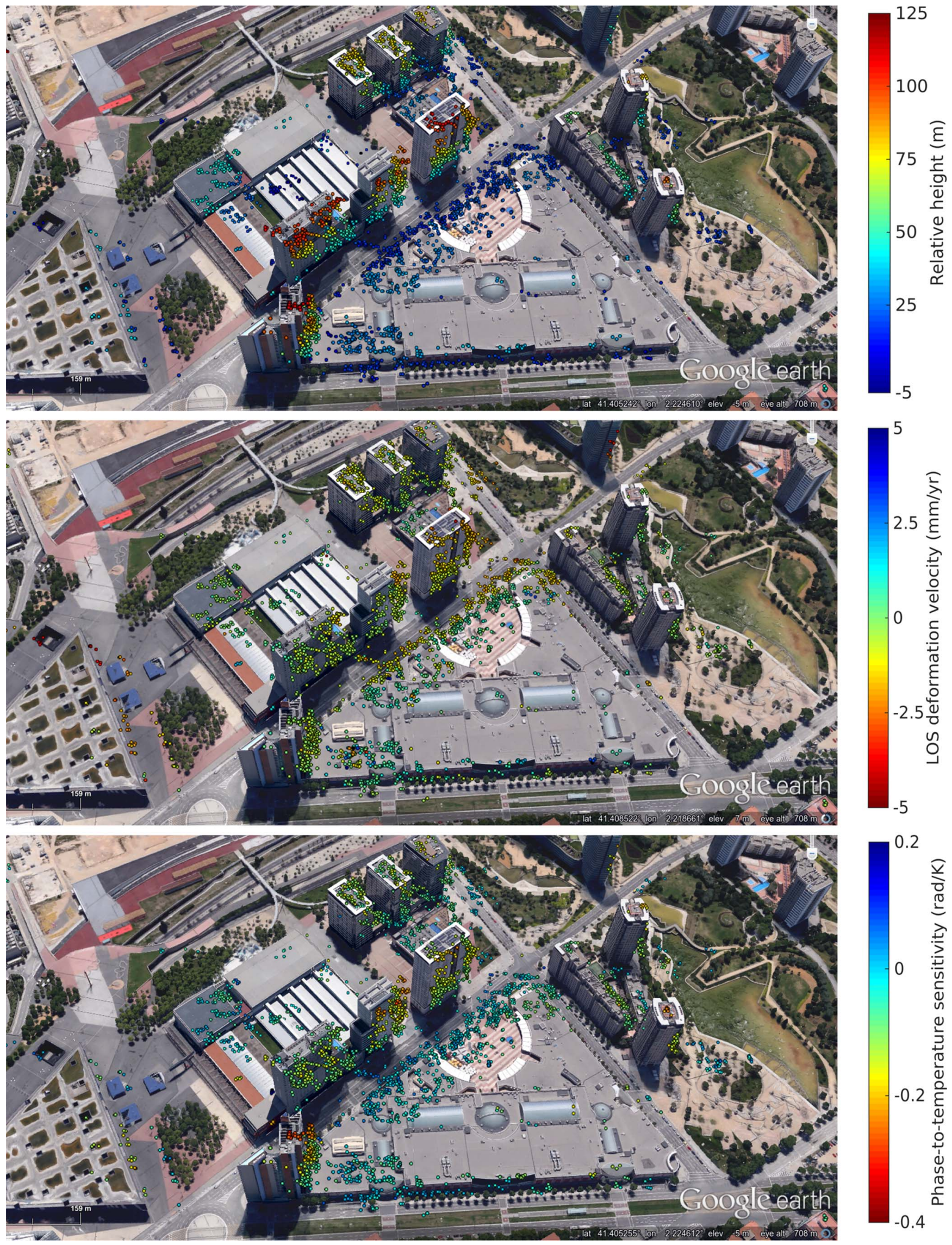

Fig. 10. Double scatterers obtained with tomographic inversion and SGLRTC, and projected on Google Earth 3-D building models. The thresholds for detection $T_{1}$ and $T_{2}$ are set at 0.4 . The colors represent the estimated parameters. (Top row) Relative height. (Middle row) Average deformation velocity in LOS. (Bottom row) Phase-to-temperature sensitivity. 

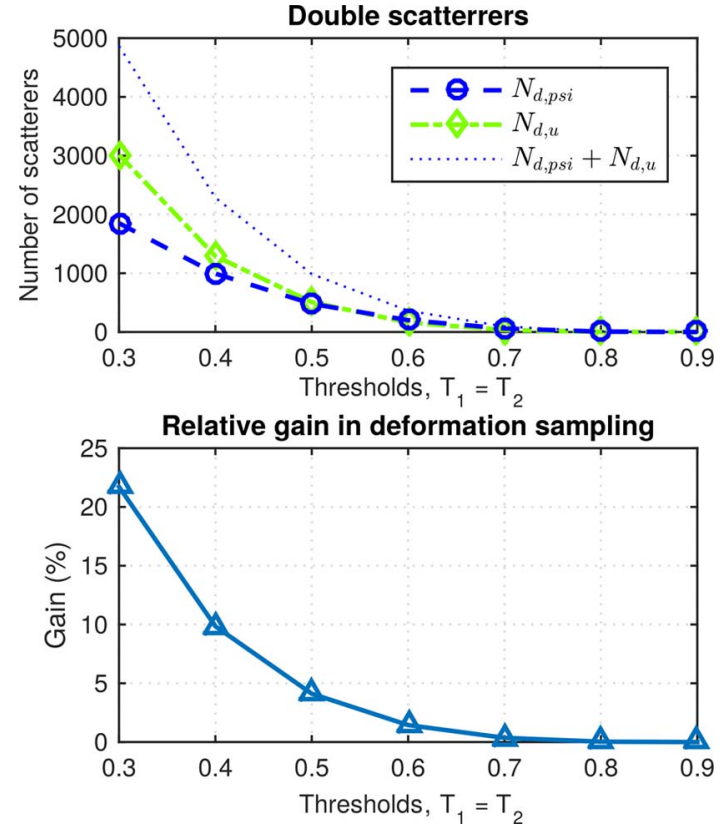

Fig. 11. Top: Number of pixels detected as double scatterers in the Diagonal Mar area at different thresholds of detection: $N_{d, \text { psi }}$ are the double scatterers for which the dominant (coherent) scatterer was also detected as a PS, whereas $N_{d, u}$ are the remaining double scatterers that were totally rejected during the IPTA-based PSI processing. Below: Corresponding relative gain in the deformation sampling, $G[(33)]$ with layover separations.

3) Double Scatterers Obtained With Tomography: Fig. 14 shows the distribution of the RMS phase deviation, $\sigma^{\text {tomo }}$ for the double scatterers obtained with tomography at different detection thresholds. There is a tradeoff between the quality of the scatterers and the relative gain in deformation sampling, $G$ (shown in Fig. 11). For $T_{1}=T_{2}=0.40$, around $99 \%$ of the detected double scatterers have $\sigma^{\text {tomo }}$ less than $1.1 \mathrm{rad}$, with a gain of $9.8 \%$, whereas the average $\sigma^{\text {tomo }}$ is $0.66 \mathrm{rad}$.

For a pixel that is truly a double scatterer, we expect that modeling the second scatterer after the first should lead to an improvement in quality in terms of reduced phase deviation between the measurements and the model fit. To this end, we define the relative decrease in the RMS phase deviation $\triangle \sigma$ as follows:

$$
\triangle \sigma=\frac{\left.\left.\sigma^{\text {tomo }}\right\rfloor_{\mathcal{H}^{1}}-\sigma^{\text {tomo }}\right\rfloor_{\mathcal{H}^{2}}}{\left.\sigma^{\text {tomo }}\right\rfloor_{\mathcal{H}^{1}}}
$$

where $\left.\sigma^{\text {tomo }}\right\rfloor_{\mathcal{H}^{1}}$ is the RMS phase deviation computed considering only one scatterer in the pixel, whereas $\left.\sigma^{\text {tomo }}\right\rfloor_{\mathcal{H}^{2}}$ is the RMS phase deviation when both the scatterers are modeled [refer to (24)]. Fig. 15 shows the distribution of $\triangle \sigma$ for the pixels detected as double scatterers at different thresholds. It can be seen that $\triangle \sigma$ tends to increase with increasing thresholds, implying that the quality improvement is higher at higher thresholds. Fig. 15 also shows that for a few double scatterers, $\triangle \sigma$ is undesirably negative implying a worsening of the quality. With increasing thresholds, the percentage of such pixels decreases. For the double scatterers detected at $T_{1}=T_{2}=0.4$, there is $19 \%$ (on average) improvement in the quality, whereas around $2 \%$ of the double scatterers witness a decrease in quality.

\section{E. Quantity and Quality of the Scatterers on a Single Layover-Affected Building}

Fig. 16 shows the relative gain in deformation sampling and the RMS phase deviation, $\sigma^{\text {tomo }}$ for the single and double scatterers detected on a single layover-affected building (Hilton hotel, as marked in the red outline). Again, we observe a sharp decline in the gain, and an improvement in quality, with increasing thresholds of detection. It can also be seen (in comparison with Fig. 11) that, for the same thresholds, the relative gain in deformation sampling is higher for the case of the single building than for the Diagonal Mar area in general. This is naturally expected since it is the layover-affected areas where PSI is likely to reject candidates and tomography likely to provide a relative gain by resolving the layovers. At $T_{1}=$ $T_{2}=0.4$, the relative gain is $31 \%$ for the single building as opposed to $9.8 \%$ for Diagonal Mar.

\section{DISCUSSION}

Here an itemized discussion of the results presented earlier is provided.

\section{A. PSI Solution}

The PSs identified in the IPTA-based PSI processing, and the estimated residual height, linear deformation, and the phase-totemperature sensitivity for each PS are shown in Fig. 3. The residual heights shown are relative to the WGS-84 ellipsoid. The estimated heights correspond fairly well with the actual heights of the buildings. The area is mostly stable, except for some regions which are undergoing deformation as much as 3-5 mm/yr (Forum area in the northeast). It can be seen that for most of the buildings, the estimated phase-to-temperature sensitivity increases with height, implying that the upper parts of the building tend to undergo more thermal expansion. This seems plausible since the base of the buildings is grounded and the upper parts are less constrained in terms of expansion. However, it may not be true for all buildings. The structure of the individual buildings would likely have a significant impact on their thermal expansion characteristics. In an earlier work [40], the estimated phase-to-temperature sensitivities were related to the linear expansion coefficient of the building material.

It can be seen in Fig. 3 that the deformation sampling achieved with the PSI processing is fairly reasonable. Even for the layovers (high-rise buildings), the PSI solution provides decent coverage. In case of a layover-affected pixel, quite often, only one of the superposing scatterers behaves coherently over time, and when it tends to dominate the other scatterer(s) in terms of energy, the pixel may still be well identified as a PS. In the case that the energy of the superposing scatterers is comparable and they exhibit temporal phase stability, the pixel would be rejected in the PSI processing, but tomography would be able to individually detect them and thus provide an added value.

\section{B. Tomographic Inversion and Scatterer Detection}

1) Layover-Affected Pixel: The results obtained with classical SAR tomography [P1, (2)] and differential tomography 


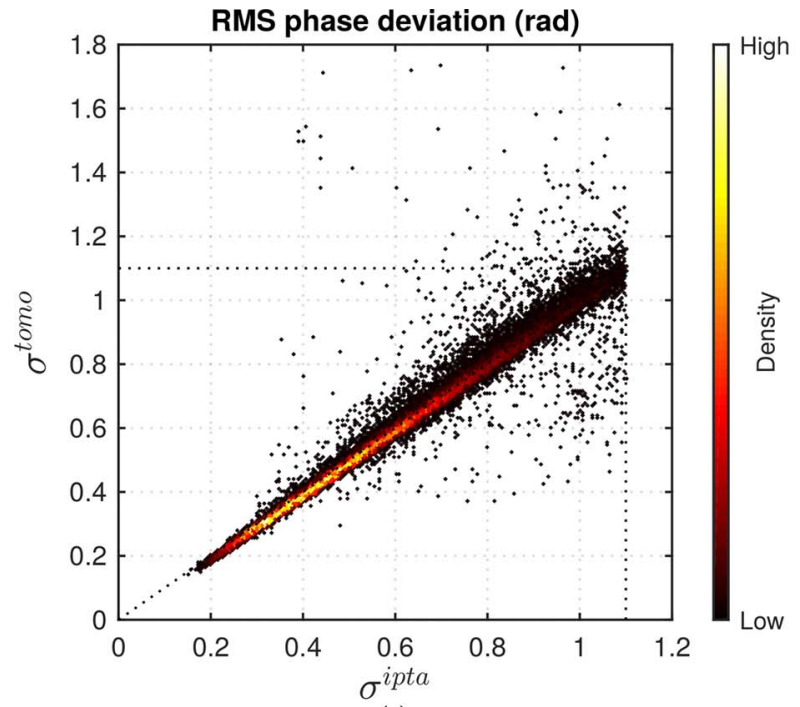

(a)

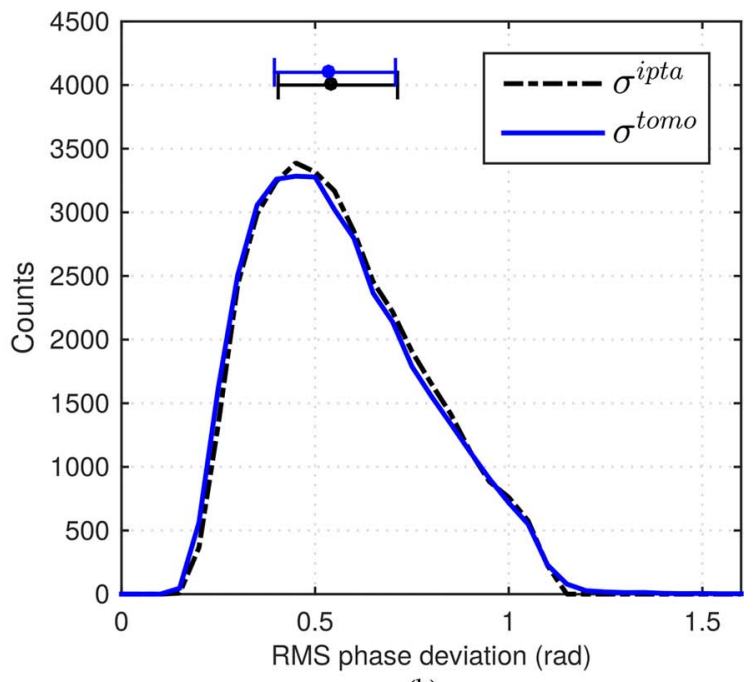

(b)

Fig. 12. Comparison of the RMS phase deviations separately obtained with PSI $\sigma^{\text {ipta }}$ and with tomography $\sigma^{\text {tomo }}$ for the pixels identified as the PSs in the IPTAbased PSI processing. (a) Joint distribution of the RMS phase deviations. The color represents the joint density. (b) Marginal histograms of the RMS phase deviations.

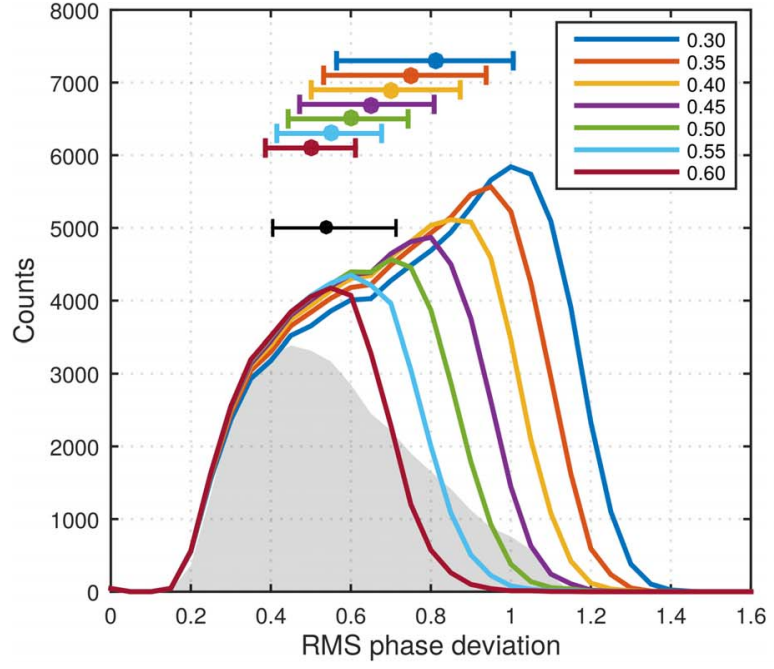

(a)

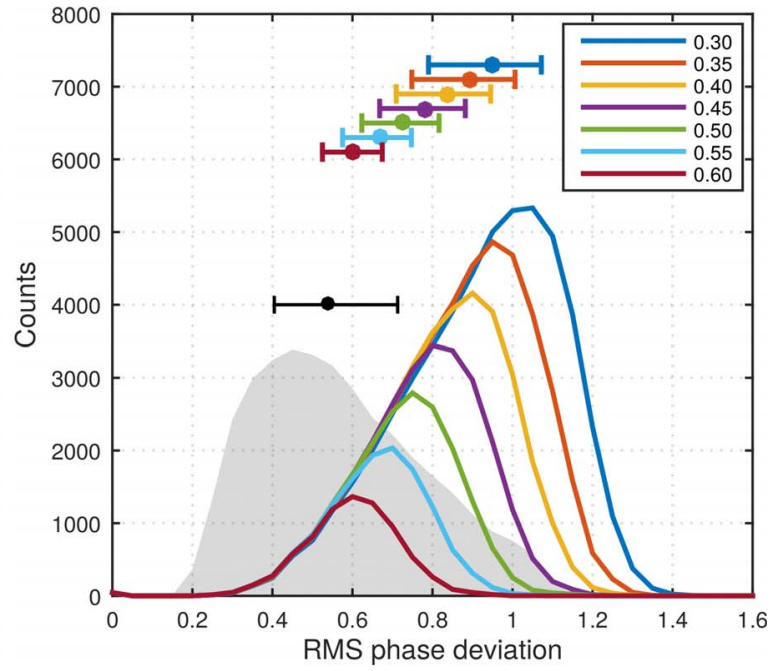

(b)

Fig. 13. Histograms of the RMS phase deviation $\sigma^{\text {tomo }}$ for (a) scatterers detected as single scatterers and (b) scatterers detected as single scatterers in addition to the PSs identified with IPTA-based PSI processing, at different thresholds (shown in different colors). The deviation bars show the interquartile range.

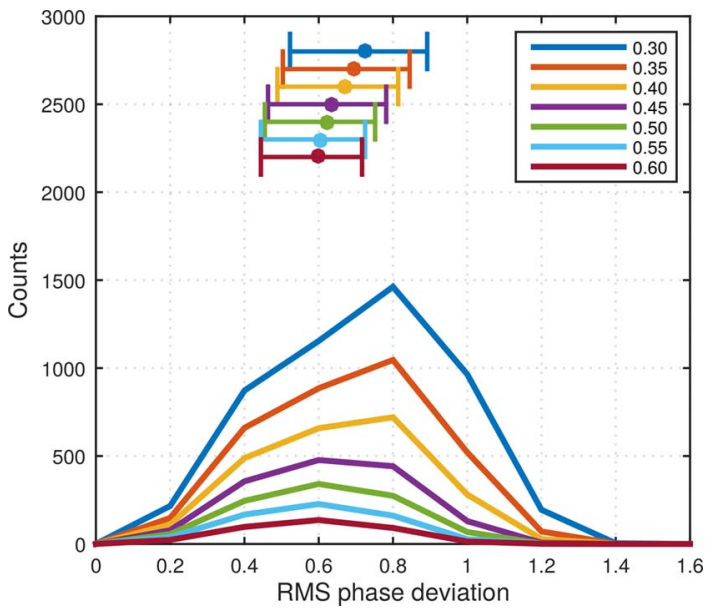

Fig. 14. Histograms of the RMS phase deviation $\sigma^{\text {tomo }}$ for double scatterers obtained with tomography, at different detection thresholds (shown in different colors). The deviation bars show the interquartile range.
$[\mathrm{P} 2,(4)]$ are reasonable in their own right but incorrect as they lead to the false inference that the pixel contains a single dominant scatterer (the first scatterer). The layover is resolved only for the extended phase model [P3, (6)] when the scatterer elevation, linear deformation, and thermal expansion are modeled simultaneously. It implies that modeling the thermal expansion of the scatterers, in addition to their elevation and linear deformation, is indeed critical for effective layover separation, particularly in the case of high-rise buildings. The estimated heights of the two individual scatterers correspond fairly accurately with the actual heights of the two buildings. In addition, we obtain estimates of the linear deformation velocity as well as the phase-to-temperature sensitivity for each of the two scatterers. Considering the pixel was originally rejected during the PSI analysis, the layover separation provides two additional deformation samples. In this way, SAR tomography provides a value-addition to the PSI analysis by improving deformation analysis in layover-affected areas. 

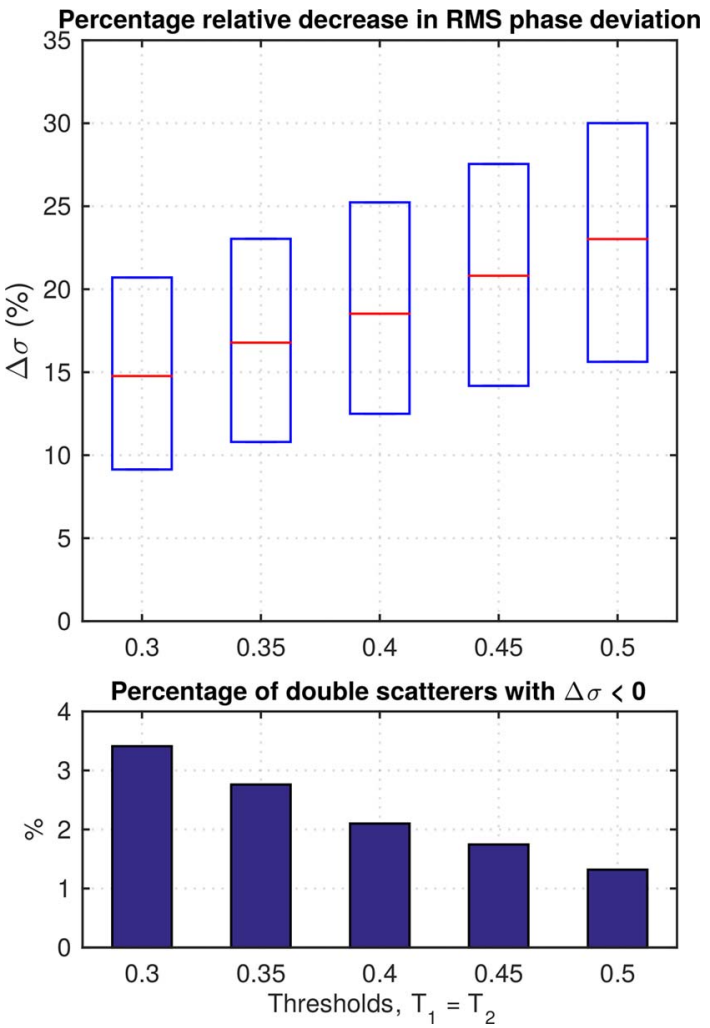

Fig. 15. (Top) Distribution of the relative decrease in RMS phase deviation, $\triangle \sigma$ when a second scatterer is modeled in addition to the first scatter, for the pixels detected as double scatterers at different thresholds. The boxplots (blue) enclose the interquartile range, and the center line (red) marks the median value. (Below) The percentage of double scatterers for which additionally modeling the second scatterer led to an increase in the RMS phase deviation, possibly representing false alarms.

2) Diagonal Mar: For thresholds below 0.3, the sudden jump in the number of double scatterers alongside the sharp decline in the gradient for single scatterers, can be explained in terms of the decision strategy adopted in (22) and (23). The SGLRTC first decides whether a given pixel is a double scatterer. If the threshold $T_{2}$ is too low, many potential single scatterers would be falsely classified as double scatterers before being explicitly tested as single scatterers. Therefore, in our case, it is appropriate to choose thresholds higher than 0.3 . The scatterers detected at $T_{1}=T_{2}=0.4$ are as shown in Figs. 8-10. It can be seen that their 3-D positions fit well to the Google Earth buildings models. The retrieved heights are fairly accurate. As with the PSI results, we observe some deformation in the Forum area. The upper parts of the buildings tend to be more sensitive to temperature-dependent phase variations.

\section{Relative Gain in Deformation Sampling With Layover Separations}

It can be seen in Fig. 11 that among the total pixels detected as double scatterers with tomography (for thresholds greater than 0.4), nearly half of them have also been identified as PSs in the IPTA-based PSI processing. It clearly indicates that tomography has improved the deformation sampling not only by resolving those layovers which were altogether rejected in the PSI processing but also by detecting the second scatterer for those where the first (dominant) was identified as a PS. The
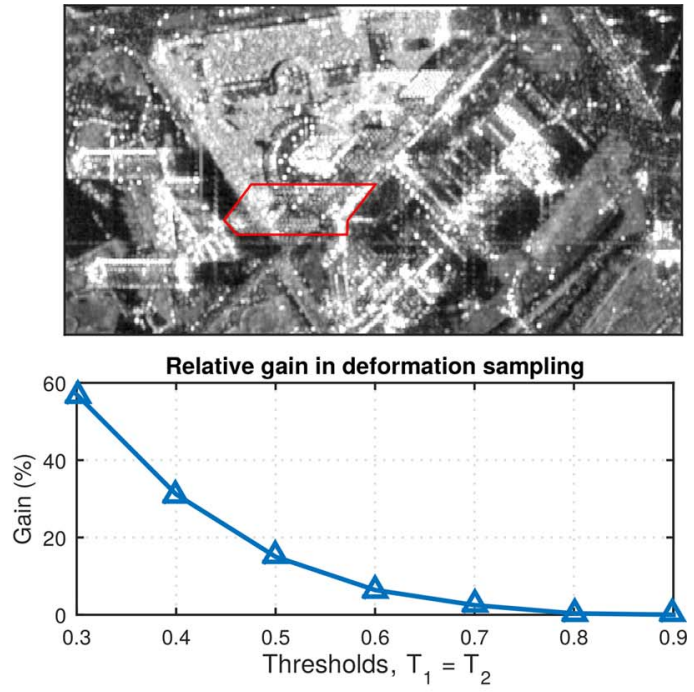

RMS phase deviation for the scatterers detected

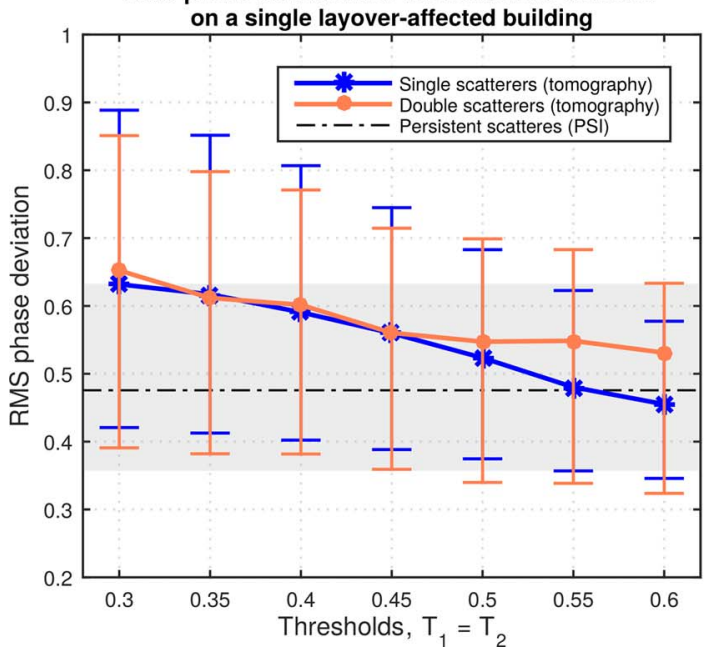

Fig. 16. Quantity and quality of the scatterers obtained with tomography on a single layover-affected building. (Top) Average SAR backscatterer of the selected building (outlined in red). (Middle) Relative gain in deformation sampling compared to a PSI solution. (Bottom) The quality of the detected single and double scatterers expressed in terms of RMS phase deviation, $\sigma^{\text {tomo }}$. The vertical bars and the shaded gray region represent the interquartile ranges for scatterers obtained with tomography and PSI, respectively.

relative gain in deformation sampling, $G$ [see (33)] declines sharply as the thresholds are increased due to a sharp decrease in the number of double scatterers. For thresholds between $0.3-0.4$, the gain varies between $22-9.8 \%$; at 0.6 , the gain is already below $1.5 \%$. In order to choose a suitable range for the thresholds, such that they are neither too restrictive nor too loose, it is imperative to consider the corresponding quality of the estimated parameters.

\section{Quality of the Estimates}

1) PSs Identified With IPTA-Based PSI Processing: The average $\sigma^{\text {ipta }}$ for the entire set of pixels identified as PSs is $0.57 \mathrm{rad}$, which is well below the upper limit of $1.1 \mathrm{rad}$ set in the IPTA processing, implying the overall good quality of the estimates obtained with PSI. For these pixels, the corresponding quality of the estimates computed with tomography is comparable, as indicated by the strong correlation between the $\sigma^{\text {ipta }}$ and $\sigma^{\text {tomo }}$ (shown in Fig. 12). 
2) Single Scatterers Obtained With Tomography: It it shown in Fig. 13(a) that progressively increasing the thresholds tends to reject the detected single scatterers of relatively lower quality, whereas those with higher quality seem mostly unaffected. In this sense, the threshold $T_{1}$ can be considered a proxy for the quality of single scatterers. In order that the quality of these scatterers is comparable with the quality of the PSs, we need thresholds greater than 0.5. However, as discussed earlier, for thresholds above 0.5 , we detect very few double scatters, and the relative gain in deformation sampling is merely $4 \%$ or less. Similarly, if we only observe the single scatterers in addition to PSs, as shown in Fig. 13(b), to match their quality with those of the PSs would require thresholds that are too restrictive to allow for the detection of a reasonable number of double scatterers.

3) Double Scatterers Obtained With Tomography: Fig. 14 shows the quality of the double scatterers detected at different thresholds. As for the case of single scatterers, there is a tradeoff between the quality and the quantity of the double scatterers. However, it can be seen that the effect of progressively increasing the thresholds on the average quality is not as pronounced as it has been for the case of single scatterers. With increasing thresholds, some pixels of relatively good quality are also rejected. This is not unexpected since the phase residue for a double scatterer depends on the quality of both the first and the second scatterer. It could be that the second scatterer suffers from phase noise leading to poor inversion; a high value of $T_{2}$ may reject it as a double scatterer, although it may still exhibit a relatively low $\sigma^{\text {tomo }}$ due to a possibly good inversion of the first scatterer. At the same time, there is a pronounced relative increase in the quality by modeling the second scatterer in addition to the first, as depicted by the increasing $\triangle \sigma$ with increasing thresholds in Fig. 15. The pixels detected as double scatterers but with $\triangle \sigma<0$ are possibly false alarms; the percentage of these pixels tends to decrease with increasing thresholds.

\section{E. Relative Gain and Quality of the Scatterers for a Single Layover-Affected Building}

In Fig. 16, we can clearly observe the tradeoff between the quantity and the quality of the scatterers for the case of a single (layover-affected) building as well. In order that the quality of the single scatterers obtained with tomography is on a par with the quality of the PSs identified with IPTA, we require $T_{1}=T_{2}>=0.55$, which allows in this case around $10 \%$ gain in deformation sampling relative to the PSI solution. For thresholds above 0.6 , we have too few detections to compute the statistics. Keeping the thresholds at 0.4 would allow as much as $31 \%$ gain but, of course, with a compromise in quality.

\section{CONCLUSION}

In this paper, we have performed a case study on the usefulness of single-look SAR tomography as an add-on to PSI for improving deformation analysis in urban areas using an interferometric stack of 50 TerraSAR-X images of an urban locality in Barcelona, Spain. Stripmap-mode images have been used in this paper, keeping in view their wider prevalence compared with higher resolution imaging modes that are normally oper- ated for a limited number of sites. A PSI analysis is performed using the IPTA framework, and is followed by tomographic inversion on the phase-calibrated stack employing a single-look BF-based merit function. The phase models for classical SAR tomography (3-D SAR), differential tomography, and the one further extended to simultaneously model thermal expansion, are compared against each other with respect to their suitability in resolving layovers. The results obtained confirm that modeling thermal expansion of the scatterers, in addition to linear deformation and elevation, is indeed critical for effective layover separations, especially in the case of high-rise buildings.

The PSI solution obtained with the IPTA framework offers a fairly reasonable coverage. Even in layover areas, many pixels have been identified as PSs (where one among the superposing scatterers tends to dominate the others and exhibits long term coherence). At the same time, there is a significant number of layover-affected pixels along the facades of the high-rise buildings which have been either totally rejected in the PSI processing, or partially rejected in the sense that a second coherent scatterer is present but has not been individually identified as a PS (since a PS is by definition a single dominant scatterer). Tomographic inversion effectively resolves these layovers and thus prevents the aforementioned rejections. Therefore, while PSI suffices for a large-scale deformation analysis, SAR tomography proves useful in performing a small-scale analysis for local infrastructure by increasing deformation samples in layoveraffected areas.

We have used a GLRT-based hypothesis testing (following the tomographic inversion) to detect single and double scatterers in the observed scene. The impact of the detection thresholds on both the quantity and the quality of the detected scatterers has also been studied, and the relative gain in deformation sampling with layover separations is computed at different thresholds. The quality of the detected scatterers is assessed in terms of the RMS phase deviation between the measurements (SLC values) and the tomographic model fit, which is consistent with the way the quality of the PSs is evaluated in the IPTAbased PSI processing. The results obtained highlight a tradeoff between the quantity and the quality; therefore, the choice of the detection thresholds has to be determined as a compromise between the desired values of the relative gain in deformation sampling and the corresponding quality. In order that the quality of the overall detected scatterers is comparable to the quality of the PSs identified with the PSI processing, the detection process may become too restrictive to allow for a sufficient number of layover separations. With a moderate compromise, we obtain a relative gain in deformation sampling of $9.8 \%$ for the Diagonal Mar area, whereas the RMS phase deviation for $99 \%$ of the detected double scatterers and single scatterers (in addition to the PSs) is below $1.1 \mathrm{rad}$ (which is set as the upper limit to allow for the acceptance of a PSC in the final PSI solution). For the double scatterers, the relative decrease in the RMS phase deviation by modeling a second scatterer in addition to the first is $19 \%$ on average. This improvement in quality clearly indicates that even in case the first scatterer was identified as a PS with a PSI approach, SAR tomography additionally allows detecting the second scatterer and thereby offers an improvement in the quality. 


\section{REFERENCES}

[1] G. Vilardo, R. Isaia, G. Ventura, P. De Martino, and C. Terranova, "InSAR permanent scatterer analysis reveals fault re-activation during inflation and deflation episodes at Campi Flegrei caldera," Remote Sens. Environ., vol. 114, no. 10, pp. 2373-2383, Oct. 2010.

[2] A. Hooper, P. Segall, and H. Zebker, "Persistent scatterer interferometric synthetic aperture radar for crustal deformation analysis, with application to Volcán Alcedo, Galápagos,” J. Geophys. Res. Solid Earth, vol. 112, no. B7, Jul. 2007, Art. no. B07407.

[3] R. Lanari, F. Casu, M. Manzo, and P. Lundgren, "Application of the SBAS-DInSAR technique to fault creep: A case study of the Hayward fault, California," Remote Sens. Environ., vol. 109, no. 1, pp. 20-28, Jul. 2007.

[4] S. Bianchini et al., "Multitemporal evaluation of landslide movements and impacts on buildings in San Fratello (Italy) by means of C-Band and X-Band PSI data," Pure Appl. Geophys., vol. 172, no. 11, pp. 3043-3065, Apr. 2014.

[5] M. Crosetto, J. A. Gili, O. Monserrat, M. Cuevas-González, J. Corominas, and D. Serral, "Interferometric SAR monitoring of the Vallcebre landslide (Spain) using corner reflectors," Natural Hazards Earth Syst. Sci., vol. 13, no. 4, pp. 923-933, 2013.

[6] B. Yu et al., "Subsidence detection by TerraSAR-X interferometry on a network of natural persistent scatterers and artificial corner reflectors," Comput. Geosci., vol. 58, pp. 126-136, Aug. 2013.

[7] G. Herrera, R. Tomàs, F. Vicente, J. Lopez-Sanchez, J. Mallorquì, and J. Mulas, "Mapping ground movements in open pit mining areas using differential SAR interferometry," Int. J. Rock Mech. Mining Sci., vol. 47, no. 7, pp. 1114-1125, Oct. 2010.

[8] R. Tomàs, G. Herrera, J. Delgado, J. Lopez-Sanchez, J. Mallorquì, and J. Mulas, "A ground subsidence study based on DInSAR data: Calibration of soil parameters and subsidence prediction in Murcia City (Spain)," Eng. Geol., vol. 111, no. 1-4, pp. 19-30, Feb. 2010.

[9] T. Strozzi, U. Wegmuller, C. Werner, A. Wiesmann, and V. Spreckels, "JERS SAR interferometry for land subsidence monitoring," IEEE Trans. Geosci. Remote Sens., vol. 41, no. 7, pp. 1702-1708, Jul. 2003.

[10] A. Gabriel, R. Goldstein, and H. Zebker, "Mapping small elevation changes over large areas: Differential radar interferometry," J. Geophys. Res., Solid Earth, vol. 94, no. B7, pp. 9183-9191, Jul. 1989.

[11] D. Massonnet and T. Rabaute, "Radar interferometry: Limits and potential," IEEE Trans. Geosci. Remote Sens., vol. 31, no. 2, pp. 455-464, Mar. 1993

[12] A. Ferretti, C. Prati, and F. Rocca, "Permanent scatterers in SAR interferometry," IEEE Trans. Geosci. Remote Sens., vol. 39, no. 1, pp. 8-20, Jan. 2001.

[13] C. Werner, U. Wegmüller, T. Strozzi, and A. Wiesmann, "Interferometric point target analysis for deformation mapping," in Proc. IEEE Int. Geosci. Remote Sens. Symp., 2003, pp. 4362-4364.

[14] P. Blanco-Sánchez, J. J. Mallorquí, S. Duque, and D. Monells, "The Coherent Pixels Technique (CPT): An advanced DInSAR technique for nonlinear deformation monitoring," Pure Appl. Geophys., vol. 165, pp. 1167-1193, Aug. 2008.

[15] A. Hooper, H. Zebker, P. Segall, and B. Kampes, "A new method for measuring deformation on volcanoes and other natural terrains using InSAR persistent scatterers," Geophys. Res. Lett., vol. 31, no. 23, Dec. 2004, Art. no. L23611.

[16] B. M. Kampes and N. Adam, "The STUN algorithm for persistent scatterer interferometry," in Proc. FRINGE Workshop, 2006, vol. 610, pp. $1-14$.

[17] M. Crosetto, O. Monserrat, R. Iglesias, and B. Crippa, "Persistent scatterer interferometry: Potential, limits and initial C- and X-band comparison," Photogramm. Eng. Remote Sens., vol. 76, no. 9, pp. 1061-1069, 2010.

[18] N. Adam, A. Parizzi, M. Eineder, and M. Crosetto, "Practical persistent scatterer processing validation in the course of the Terrafirma project," J. Appl. Geophys., vol. 69, no. 1, pp. 59-65, Sep. 2009.

[19] S. Gernhardt, N. Adam, M. Eineder, and R. Bamler, "Potential of very high resolution SAR for persistent scatterer interferometry in urban areas," Ann. GIS, vol. 16, pp. 103-111, Aug. 2010.

[20] A. Ferretti, M. Bianchi, C. Prati, and F. Rocca, "Higher-order permanent scatterers analysis," EURASIP J. Adv. Signal Process., vol. 2005, no. 20, pp. 3231-3242, 2005.

[21] A. Reigber and A. Moreira, "First demonstration of airborne SAR tomography using multibaseline L-Band data," IEEE Trans. Geosci. Remote Sens., vol. 38, no. 5, pp. 2142-2152, Sep. 2000.

[22] F. Gini, F. Lombardini, and M. Montanari, "Layover solution in multibaseline SAR interferometry," IEEE Trans. Aerosp. Electron. Syst., vol. 38, no. 4, pp. 1344-1356, Oct. 2002.
[23] G. Fornaro, F. Serafino, and F. Soldovieri, "Three-dimensional focusing with multipass SAR data," IEEE Trans. Geosci. Remote Sens., vol. 41, no. 3, pp. 507-517, Mar. 2003.

[24] O. Frey and E. Meier, "3-D time-domain SAR imaging of a forest using airborne multibaseline data at L-and P-bands," IEEE Trans. Geosci. Remote Sens., vol. 49, no. 10, pp. 3660-3664, Oct. 2011

[25] O. Frey, I. Hajnsek, U. Wegmüller, and C. Werner, "SAR tomography based 3-D point cloud extraction of point-like scatterers in urban areas," in Proc. IEEE Int. Geosci. Remote Sens. Symp., 2014, pp. 1313-1316.

[26] G. Fornaro, F. Lombardini, A. Pauciullo, D. Reale, and F. Viviani, "Tomographic processing of interferometric SAR data: Developments, applications, and future research perspectives," IEEE Signal Process. Mag., vol. 31, no. 4, pp. 41-50, Jul. 2014.

[27] F. Lombardini, M. Montanari, and F. Gini, "Reflectivity estimation for multibaseline interferometric radar imaging of layover extended sources," IEEE Trans. Signal Process., vol. 51, no. 6, pp. 1508-1519, Jun. 2003.

[28] F. Lombardini, F. Cai, and D. Pasculli, "Spaceborne 3-D SAR tomography for analyzing garbled urban scenarios: Single-look superresolution advances and experiments," IEEE J. Sel. Topics Appl. Earth Observ. Remote Sens., vol. 6, no. 2, pp. 960-968, Apr. 2013.

[29] X. Zhu and R. Bamler, "Superresolving SAR tomography for multidimensional imaging of urban areas: Compressive sensing-based TomoSAR inversion,” IEEE Signal Process. Mag., vol. 31, no. 4, pp. 51-58, Jul. 2014.

[30] X. Zhu and R. Bamler, "Very high resolution spaceborne SAR tomography in urban environment," IEEE Trans. Geosci. Remote Sens., vol. 48, no. 12, pp. 4296-4308, Dec. 2010.

[31] F. Lombardini, "Differential tomography: A new framework for SAR interferometry," IEEE Trans. Geosci. Remote Sens., vol. 43, no. 1, pp. 37-44, Jan. 2005 .

[32] F. Lombardini, "Differential tomography: A new framework for SAR interferometry," in Proc. IEEE Int. Geosci. Remote Sens. Symp., 2003, vol. 2, pp. 1206-1208.

[33] G. Fornaro, F. Reale, and D. Serafino, "Four-dimensional SAR imaging for height estimation and monitoring of single and double scatterers," IEEE Trans. Geosci. Remote Sens., vol. 47, no. 1, pp. 224-237, Jan. 2009.

[34] A. De Maio, G. Fornaro, and A. Pauciullo, "Detection of single scatterers in multidimensional SAR imaging," IEEE Trans. Geosci. Remote Sens., vol. 47, no. 7, pp. 2284-2297, Jul. 2009.

[35] X. Zhu and R. Bamler, "Compressive sensing for high resolution differential SAR tomography-The SL1MMER algorithm," in Proc. IEEE Int. Geosci. Remote Sens. Symp., 2010, pp. 17-20.

[36] D. Reale, G. Fornaro, A. Pauciullo, X. Zhu, and R. Bamler, "Tomographic imaging and monitoring of buildings with very high resolution SAR data," IEEE Geosci. Remote Sens. Lett., vol. 8, no. 4, pp. 661-665, Jul. 2011.

[37] F. Lombardini and M. Pardini, "Superresolution differential tomography: Experiments on identification of multiple scatterers in spaceborne SAR data," IEEE Trans. Geosci. Remote Sens., vol. 50, no. 4, pp. 1117-1129, Apr. 2012.

[38] A. Pauciullo, D. Reale, A. De Maio, and G. Fornaro, "Detection of double scatterers in SAR tomography," IEEE Trans. Geosci. Remote Sens., vol. 50, no. 9, pp. 3567-3586, Sep. 2012.

[39] O. Monserrat, M. Crosetto, M. Cuevas, and B. Crippa, "The thermal expansion component of persistent scatterer interferometry observations," IEEE Geosci. Remote Sens. Lett., vol. 8, no. 5, pp. 864-868, Sep. 2011.

[40] U. Wegmuller and C. Werner, "Mitigation of thermal expansion phase in persistent scatterer interferometry in an urban environment," in Proc. Joint Urban Remote Sens. Event, Mar. 2015, pp. 1-4.

[41] X. Zhu and R. Bamler, "Let's do the time warp: Multicomponent nonlinear motion estimation in differential SAR tomography," IEEE Geosci. Remote Sens. Lett., vol. 8, no. 4, pp. 735-739, Jul. 2011.

[42] Y. Wang, X. Zhu, and R. Bamler, "An efficient tomographic inversion approach for urban mapping using meter resolution SAR image stacks," IEEE Geosci. Remote Sens. Lett., vol. 11, no. 7, pp. 1250-1254, Jul. 2014.

[43] M. Siddique, I. Hajnsek, U. Wegmüller, and O. Frey, "Towards the integration of SAR tomography and PSI for improved deformation assessment in urban areas," in Proc. FRINGE Workshop, 2015, pp. 1-6.

[44] M. Siddique, I. Hajnsek, U. Wegmüller, and O. Frey, "Investigating the combined use of differential SAR tomography and PSI for spatio-temporal inversion," in Proc. Joint Urban Remote Sens. Event, 2015, pp. 1-4.

[45] D. Reale, G. Fornaro, and A. Pauciullo, "Extension of 4-D SAR imaging to the monitoring of thermally dilating scatterers," IEEE Trans. Geosci. Remote Sens., vol. 51, no. 12, pp. 5296-5306, Dec. 2013.

[46] O. Frey, F. Morsdorf, and E. Meier, "Tomographic imaging of a forested area by airborne multi-baseline P-Band SAR," Sensors, vol. 8, no. 9, pp. 5884-5896, Sep. 2008.

[47] O. Frey and E. Meier, "3D SAR imaging of a forest using airborne MB-SAR data at L-and P-band: Data processing and analysis," in Proc. Euro. Conf. SAR, 2010, pp. 1-4. 
[48] F. Gini and F. Lombardini, "Multibaseline cross-track SAR interferometry: A signal processing perspective," IEEE Aerosp. Electron. Syst. Mag., vol. 20, no. 8, pp. 71-93, Aug. 2005.

[49] G. Fornaro, A. Pauciullo, D. Reale, and S. Verde, "Multilook SAR tomography for 3-D reconstruction and monitoring of single structures applied to COSMO-SKYMED data," IEEE J. Sel. Topics Appl. Earth Observ. Remote Sens., vol. 7, no. 7, pp. 2776-2785, Jul. 2014.

[50] G. Fornaro, S. Verde, D. Reale, and A. Pauciullo, "CAESAR: An approach based on covariance matrix decomposition to improve multibaselinemultitemporal interferometric SAR processing," IEEE Trans. Geosci. Remote Sens., vol. 53, no. 4, pp. 2050-2065, Apr. 2015.

[51] O. Frey, I. Hajnsek, and U. Wegmuller, "Spaceborne SAR tomography in urban areas," in Proc. IEEE Int. Geosci. Remote Sens. Symp., Jul. 2013, pp. 69-72.

[52] G. Fornaro, F. Lombardini, and F. Serafino, "Three-dimensional multipass SAR focusing: Experiments with long-term spaceborne data," IEEE Trans. Geosci. Remote Sens., vol. 43, no. 4, pp. 702-714, Apr. 2005.

[53] X. Zhu and R. Bamler, "Super-resolution power and robustness of compressive sensing for spectral estimation with application to spaceborne tomographic SAR," IEEE Trans. Geosci. Remote Sens., vol. 50, no. 1, pp. 247-258, Jan. 2012.

[54] X. Zhu and R. Bamler, "Tomographic SAR inversion by $L_{1}$-norm regularization-The compressive sensing approach," IEEE Trans. Geosci. Remote Sens., vol. 48, no. 10, pp. 3839-3846, Oct. 2010.

[55] D. Massonnet and K. Feigl, "Radar interferometry and its application to changes in the Earth's surface," Rev. Geophys., vol. 36, no. 4, pp. 441-500, Nov. 1998.

[56] F. Lombardini and F. Gini, "Model order selection in multi-baseline interferometric radar systems," EURASIP J. Adv. Signal Process., vol. 2005, pp. 3206-3219, 2005.

[57] G. Fornaro and F. Serafino, "Imaging of single and double scatterers in urban areas via SAR tomography," IEEE Trans. Geosci. Remote Sens., vol. 44, no. 12, pp. 3497-3505, Dec. 2006.

[58] C. Colesanti, A. Ferretti, F. Novali, C. Prati, and F. Rocca, "SAR monitoring of progressive and seasonal ground deformation using the permanent scatterers technique," IEEE Trans. Geosci. Remote Sens., vol. 41, no. 7, pp. 1685-1701, Jul. 2003.

[59] U. Wegmuller, "Automated terrain corrected SAR geocoding," in Proc. IEEE Int. Geosci. Remote Sens. Symp., 1999, vol. 3, pp. 1712-1714.

[60] O. Frey, M. Santoro, C. L. Werner, and U. Wegmuller, "DEM-based SAR pixel area estimation for enhanced geocoding refinement and radiometric normalization," IEEE Geosci. Remote Sens. Lett., vol. 10, no. 1, pp. 48-52, Jan. 2013

[61] U. Wegmuller, O. Frey, and C. Werner, "Point density reduction in persistent scatterer interferometry," in Proc. Eur. Conf. SAR, Apr. 2012, pp. 673-676.

[62] U. Wegmuller, D. Walter, V. Spreckels, and C. Werner, "Nonuniform ground motion monitoring with TerraSAR-X persistent scatterer interferometry," IEEE Trans. Geosci. Remote Sens., vol. 48, no. 2, pp. 895-904, Feb. 2010.

[63] C. Werner, U. Wegmuller, A. Wiesmann, and T. Strozzi, "Interferometric point target analysis with JERS-1 L-band SAR data," in Proc. IEEE Int. Geosci. Remote Sens. Symp., Jul. 2003, vol. 7, pp. 4359-4361.

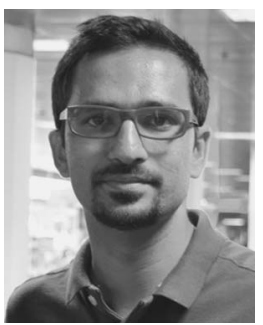

Muhammad Adnan Siddique (M'12-S'14-S'16) received the B.E. degree in electronics engineering from the National University of Sciences and Technology, Islamabad, Pakistan, in 2006 and the European Master of Research in Information and Communications Technology (MERIT) joint degree from the Karlsruhe Institute of Technology, Karlsruhe, Germany and Universitat Politcnica de Catalunya, Barcelona, Spain through an Erasmus Mundus Scholarship. He is currently working toward the Doctoral degree at the Chair of Earth Observation and Remote Sensing, Swiss Federal Institute of Technology (ETH), Zurich, Switzerland.

From 2007 to 2008, he worked as a radio-frequency (RF) planning engineer. In Fall 2010, he joined the Department of Electrical Engineering, COMSATS Institute of Information Technology, Lahore, Pakistan, as a Lecturer. His current research interests include synthetic aperture radar tomographic techniques for deformation assessment in urban areas.

Mr. Siddique received the President's Gold Medal for securing the highest cumulative GPA in his class during his undergraduate years.

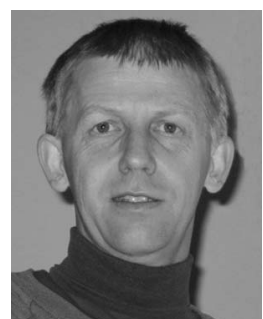

Urs Wegmüller (SM'03) received the M.S. and $\mathrm{Ph} . \mathrm{D}$. degrees in physics from the University of Bern, Bern, Switzerland, in 1986 and 1990, respectively.

Between 1991 and 1992, he was with the Jet Propulsion Laboratory, Pasadena, CA, USA, and between 1993 and 1995, with the University of Zürich, Zürich, Switzerland. In 1995, he was a Founding Member of GAMMA Remote Sensing AG (GAMMA), a Swiss company active in the development of signal processing techniques and remote sensing applications. As the Chief Executive Officer of GAMMA, he has overall responsibility for GAMMA's activities. Currently, his main involvement is in the development of applications and the definition and implementation of related services in land surface deformation mapping, hazard mapping, land use mapping, and topographic mapping. $\mathrm{He}$ is/was a Principal Investigator for projects supported by the European Space Agency and the European Commission Framework programs.

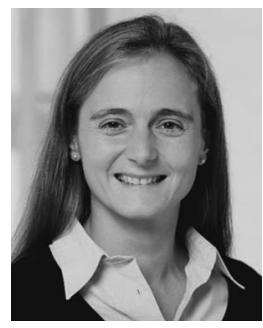

Irena Hajnsek (A'01-M'06-SM'09-F'14) received the Dipl. (Honors) degree from the Free University of Berlin, Berlin, Germany, in 1996 and the Dr. (Hons.) degree from the Friedrich Schiller University of Jena, Jena, Germany, in 2001

She is currently a Professor of Earth observation and remote sensing with the Institute of Environmental Engineering, Swiss Federal Institute of Technology (ETH) Zurich, Zurich, Switzerland, and the Head of the Polarimetric SAR Interferometry Research Group, Microwaves and Radar Institute, German Aerospace Center, Wessling, Germany. Since 2010, she has been the Science Coordinator of the German satellite mission TanDEM-X. Her main research interests are in electromagnetic propagation and scattering theory, radar polarimetry, SAR and interferometric SAR data processing techniques, and environmental parameter modeling and estimation.

Dr. Hajnsek has been a member of the IEEE Geoscience and Remote Sensing Society Administrative Committee since 2013. She served as the Technical Program Cochair of the IEEE International Symposium on Geoscience and Remote Sensing in 2012

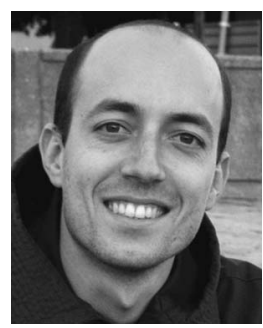

Othmar Frey (S'04-M'08) received the M.Sc (Hons.) degree in geomatic engineering from the Swiss Federal Institute of Technology (ETH), Zurich, Switzerland, in 2002, and the Dr. sc. nat. (Hons.) degree in radar remote sensing from the University of Zurich, Zurich, Switzerland, in 2010.

From 2002 to 2010, he was a Research Associate with the Remote Sensing Laboratories, University of Zurich. Since January 2011, he has been with ETH Zurich, as a Senior Research Fellow and Lecturer, and with GAMMA Remote Sensing AG, Gümligen, Switzerland, as a Senior Project Scientist. He has been active in various national and international research projects, as a Principal Investigator (PI) and Project Manager as well as a Co-Investigator, among them, the joint industry-academic project "GAMMA Software Module for Spaceborne SAR Tomography," in the role of the PI and Project Manager. At GAMMA, he has also been active in the development of the Gamma software and in technical consulting related to SAR imaging and interferometric applications. He has been a member of the Science Expert Group for ESA's SAOCOM-CS companion satellite mission since 2014. His research interests include 2-D and 3-D (tomographic) SAR focusing techniques and interferometric techniques for applications such as 3-D forest mapping, ground deformation monitoring (persistent scatterer interferometry/differential tomography), and profiling the structure of snowpacks, using spaceborne, airborne, and ground-based radar sensors.

Dr. Frey has been serving as the Chair of the Swiss Chapter of the IEEE Geoscience and Remote Sensing Society since 2015. He received the Distinction Award and Prize of the Faculty of Science, University of Zurich, for his Ph.D. thesis, in 2010 and the ETH medal for an outstanding M.Sc. thesis in 2002. He also received the Third Place Student Paper Award at the 2010 EUSAR Conference in Aachen, Germany. He was a corecipient of the Best Paper Award at the ISPRS Workshop Laserscanning and Silvilaser 2007 in Helsinki, Finland. 